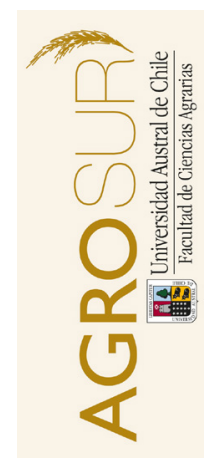

\title{
Efecto del escaldado y la temperatura en la cinética de secado de las hojas de quinua (Chenopodium quinoa Willd.), variedad Salcedo INIA
}

\author{
Effect of blanching and temperature on the drying kinetic of the \\ leaves of quinoa (Chenopodium quinoa Willd.), Salcedo INIA variety
}

\author{
Coloma-Paxi, A. ${ }^{a}$, Paquita-Ninaraqui, R. ${ }^{a}$, Velásquez-Huallpa, R. ${ }^{a}$, Abarca, R.L. ${ }^{b *}$
}

${ }^{a}$ Departamento de Agroindustrias, Facultad de Ciencias Agrarias, Universidad Nacional del Altiplano de Puno, Av. Floral 1153, Puno, Perú.

${ }^{b}$ Instituto de Ciencia y Tecnología de los Alimentos, Facultad de Ciencias Agrarias, Universidad Austral, Avda. Julio Sarrazín s/n, Isla Teja, Valdivia, Chile.

\begin{tabular}{l} 
A R T I C L E I N F O \\
\hline Keywords: \\
Quinoa leaves \\
Pre-treatment \\
Drying kinetics \\
Moisture diffusivity \\
Activation energy \\
Fitting \\
Rehydration
\end{tabular}

Original Research Article, Food Processing and Quality

*Corresponding author:

Romina Abarca Oyarce

E-mail address:

romina.abarca@uach.cl

\begin{abstract}
A B S T R A C T
The aim of this research was to compare the effects of pre-treatment (blanched in water and blanched in $3 \% \mathrm{NaCl}$ solution) respect to control and drying temperatures $\left(50,60\right.$ and $\left.70{ }^{\circ} \mathrm{C}\right)$ on quinoa leaves (Chenopodium Quinoa Willd.) variety Salcedo INIA. The study was carried out in a fixed bed dryer with continuous weight control. The parameters evaluated were the proximal chemical composition, the drying kinetics and the rehydration ratio of the leaves. The proximal contents of the samples blanched in $3 \% \mathrm{NaCl}$ solution did not show significant differences $\mathrm{P}<0.05$ with respect to the control in the content of fat, ash, fiber and protein. The drying rates of the control samples showed decreasing speeds curves, while the pre-treated, constant speeds. The experimental data were adjusted to the models of Henderson and Pabis, Midilli, Logarithmic, Page and Lewis; evaluating their validity with high values of $\mathrm{R}^{2}$, reduced by RMSE and $\chi^{2}$. The models of Midilli and Page were those that characterize the drying curves of quinoa leaves with coefficients of determination $\mathrm{R}^{2}$ high 0.9995-0.9985 with values of RMSE and $\chi^{2}$ low $0.0053-0.016$ and $4.01 \times 10^{-5}-1.23 \times 10^{-4}$, respectively. The effective diffusivity of water varied from $3.871 \times 10^{-12}$ to $1.175 \times 10^{-11} \mathrm{~m}^{2} \mathrm{~s}^{-1}$ with activation energies of 32.66 to $56.044 \mathrm{~kJ} \mathrm{~mol}^{-1}$. The rehydration ratio of the samples blanched in water and dried at $50{ }^{\circ} \mathrm{C}$ presented the best index. The equilibrium moisture reached by the quinoa leaves, after the application of the pre-treatments varied between 388 to 135 minutes.
\end{abstract}

\section{RESUMEN}

El objetivo de esta investigación fue comparar los efectos del escaldado (en agua y en solución de $\mathrm{NaCl}$ al 3\%) con respecto al control y temperaturas de secado $\left(50,60\right.$ y $70{ }^{\circ} \mathrm{C}$ ) sobre las hojas de quinua (Chenopodium Quinua Willd.) variedad Salcedo INIA. El estudio se llevó a cabo en un secador de lecho fijo con control adaptivo de peso continuo. Los parámetros evaluados fueron la composición química proximal, la cinética de secado y la relación de rehidratación de las hojas. Los contenidos proximales de las muestras escaldadas en solución de $\mathrm{NaCl}$ al 3\% no presentaron diferencias significativas $\mathrm{P}<0,05$ respecto al control en el contenido de grasa, ceniza, fibra y proteína. Las velocidades de secado de las muestras controles presentaron curvas decrecientes, mientras que las pre-tratadas, velocidades constantes. Los datos experimentales fueron ajustados a los modelos de Henderson y Pabis, Midilli, Logarítmico, Page y Lewis; evaluando su validez con altos valores de $\mathrm{R}^{2}$, reducidos de RMSE y $\chi^{2}$. Siendo los modelos de Midilli y Page los que caracterizan las curvas de secado de hojas de quinua con coeficientes de determinación $\mathrm{R}^{2}$ altos 0,9995-0,9985 con valores de RMSE y $\chi^{2}$ bajos 0,0053-0,016 y $4,01 \times 10^{-5}-1,23 \times 10^{-4}$, respectivamente. La difusividad efectiva del agua varió de $3,871 \times 10^{-12}$ a $1,175 \times 10^{11} \mathrm{~m}^{2} \mathrm{~s}^{-1} \mathrm{con}_{\text {energías }}$ de activación de 32,66 a 56,044 kJ mol-1. La relación de rehidratación de las muestras escaldadas en agua y secadas a 50 ${ }^{\circ} \mathrm{C}$ presentó el mejor índice. Los tiempos para alcanzar humedad de equilibrio en las hojas variaron de acuerdo al pretratamiento y temperatura de 388 a 135 minutos.

Palabras clave: Hojas de quinua, pre-tratamiento, cinética de secado, difusividad efectiva del agua, energía de activación, modelado, rehidratación.

\section{INTRODUCCIÓN}

La quinua (Chenopodium Quinoa Willd.) de la familia Chenoponiceae es nativa de los Andes y se ha cultivado en la Región Andina y en varias regiones de
América del Sur por varios miles de años, debido a su alta adaptabilidad a ambientes extremos, capacidad de resistir bajas temperaturas y presencia de granizos (PROINPA, 2011; Adnan Ramzani et al., 2017). La quinua es halófita, ya que es un cultivo altamente toleran- 
te a la sal (Nowak et al., 2016; Adolf et al., 2013). Es un cultivo herbáceo de $80 \mathrm{~cm}$ a $3 \mathrm{~m}$ de alto, que crece en altitudes entre 0 a 4.000 metros sobre el nivel del mar (m.s.n.m.), aunque la mejor producción se produce entre 2.500 a 3.800 m.s.n.m. y en lugares con 250$500 \mathrm{~mm}$ de precipitación anual y con temperaturas medias de 5 a $14^{\circ} \mathrm{C}$ (Mujica y Jacobsen, 2006; López et al., 2011). En los últimos años, la quinua ha adquirido una relevancia renovada debido a su excelente valor nutricional, consumido por las culturas Andinas como alimento básico. Actualmente, este pseudo-cereal se cultiva principalmente en Perú, Bolivia, Ecuador y Chile, desde donde se exporta a diferentes destinos (Navruz-Varli y Sanlier, 2016). En Europa, se encuentran pequeños cultivos. Durante las últimas dos décadas la quinua ganó gran relevancia, llegando a ser ampliamente promovida por la Organización de las Naciones Unidas para la Alimentación y la Agricultura (FAO), quien declaró el año 2013, como el año internacional de la quinua. Esto debido a sus propiedades agrícolas, sin gluten y alto valor nutricional debido a la calidad y cantidad de proteínas (en comparación con los cereales tradicionales 14-16 g $100 \mathrm{~g}^{-1}$ ) y el contenido de ácidos grasos, fibra dietética, vitaminas y minerales. Además de múltiples fotoquímicos, favoreciendo así la salud humana (Lorusso et al., 2017; Navrus-Varli y Sanlier, 2016; Vilcacund o y Hernández-Ledesma, 2017; Nowak et al., 2016). Por otra parte, las hojas de este pseudocereal, hasta ahora calificadas como desecho sin valor, son comestibles y pueden ser consumidas en ensaladas, además de ser usadas como un valioso suplemento en alimentos funcionales (Mujica y Jacobsen, 2006). Estudios realizados demostraron que el contenido de proteína que poseen las hojas de la quinua es mayor que en los granos (Cardozo y Tapia, 1979; Koziol, 1992; Schlick, 2000; Galarza, 2010). Las hojas de quinua contienen abundante cantidad de cenizas $(3,3 \%)$, fibra $(1,9 \%)$, nitratos $(0,4 \%)$, vitamina E $(2,9 \mathrm{mg} \alpha$-TE 100 $\left.\mathrm{g}^{-1}\right)$, vitamina $\mathrm{C}\left(1,2-2,3 \mathrm{~g} \mathrm{~kg}^{-1}\right)$, sodio (289 $\mathrm{mg}^{\left.-100 \mathrm{~g}^{-1}\right)}$ y proteínas (27-30 $\mathrm{g} \mathrm{kg}^{-1}$ ) (Gawlik-Dziki et al., 2013), aunque el contenido de saponina limita su aprovechamiento (Masterbroek et al., 2000; Fiallos-Jurado et al., 2016). Por otro lado, se ha reportado que las hojas de quinua poseen un alto contenido de compuestos fenólicos potencialmente biodisponibles (alrededor del $80 \%$ ), lo que las sitúa como una sustancia quimiopreventiva y anticancerígena (Repo-Carrasco et al., 2010; Gawlik-Dziki et al., 2013; Świeca et al., 2014). En base a las propiedades mencionadas, es necesario identificar cada uno de los productos y subproductos que se pueden obtener de las hojas, el tallo y el grano (Villacrés et al., 2011).

Las consideraciones mencionadas implican desarrollar condiciones óptimas para la conservación de las propiedades frescas de las hojas de quinua que por su alto contenido de humedad, las que son propicias para el deterioro químico, microbiológico y la acción enzimática (Bayindirli, 2010; Galarza, 2010), llevando a las hojas a una reducción de su vida útil luego de la cosecha (Meisami-asl y Rafiee, 2009; Akonor y Tortoe, 2014). Para evitar afectar la vida útil de los vegetales y tornarlos más estables en el tiempo, a nivel de procesamiento se torna factible la aplicación de la operación unitaria de secado. Según Espinoza (2011) el deshidratado se ha practicado desde los albores de la humanidad, el proceso extiende la vida en anaquel sin la adición de ningún preservante de naturaleza química y por otra, reduce el tamaño de los sistemas de envasado y costos asociados al transporte (Doymaz, 2012; Prasad y Prasad, 2013; Doymaz, 2014; TorregrozaEspinosa et al., 2014). El secado convencional con el aire caliente fue usado ampliamente, lo cual gradualmente ha ido evolucionando, utilizando hoy en día equipos de secado (Zhang y Long, 2017), esto debido a que en el antiguo proceso la velocidad de difusión reducida impedía un paso efectivo del agua a la superficie (Rao et al., 2005; Ratti, 2009). Sin embargo, con la aplicación de este método se han detectado algunos inconvenientes asociados a la degradación de la calidad de los alimentos sometidos, tales como hinchazón, cristalización, disminución de la capacidad de rehidratación, pérdidas de sabor, aromas y color, además de verse afectados los valores nutricionales (Fellows, 2000; Akonor y Tortoe, 2014; Karam et al., 2016). En base a las problemáticas asociadas al proceso, es que se hacen necesarias medidas paliativas, como la aplicación de pre-tratamientos previos al secado de las frutas y verduras con el fin de controlar las características indeseadas. Dentro de las que destaca el escaldado, proceso que consiste en sumergir el producto en agua caliente o exponerlo al vapor, básicamente para lograr la inactivación de las enzimas que provocan la hidrólisis de los lípidos y pardeamiento, así como también ablandar los tejidos y mejorar la transferencia de masa durante el secado (Severini et al., 2005; Jokić et al., 2009).

Por otro lado, el pre-tratamiento osmótico se realiza al sumergir las muestras en una solución hipertónica como paso previo al secado. Este proceso reduce el contenido inicial de humedad del material vía osmosis o modificando la estructura del tejido de una forma que incremente la transferencia de masa y reduzca el tiempo de secado (Rao et al., 2005; Sutar y Sutar, 2013; Akonor y Tortoe, 2014). Siendo el rehidratado, una medida del daño que ha recibido la muestra en el secado (Doymaz, 2014). Otro aspecto importante es el modelado, para lo que se han empleado varias ecuaciones empíricas para el secado laminar, usadas para estimar los tiempos de secado de muchos productos. Diversos investigadores han propuesto modelos matemáticos aplicados al secado laminar de varios productos agrícolas (Saeed et al., 2008; Meisami-asl y Rafiee, 
2009; Midilli et al., 2002; Darvishi et al., 2013; Simha y Gugalia, 2013. Entre ellos destacan los modelos de difusión, modelos simplificados, modelos de flujo laminar, y el secado selectivo de alimentos (Crank, 1975; Ratti, 2009).

Los objetivos de la presente investigación fueron, en primer lugar, estudiar el efecto de diversos pretratamientos recibidos por las hojas de quinua y de este modo analizar los efectos de la temperatura y pretratamientos en el secado de hojas de quinua variedad Salcedo INIA en determinar los cambios de composición químico proximal, caracterizar las cinéticas de secado y de rehidratación.

\section{MATERIALES Y MÉTODOS}

\section{Materia prima}

Hojas de quinua (variedad Salcedo INIA), frescas y enteras las cuales fueron recolectadas a los 75 y 90 días luego de la siembra en una granja local del distrito de Laraqueri Puno-Perú. Las muestras fueron recolectadas manualmente empleando una tijera, y luego transportada en un cooler y almacenada en refrigeración a $10^{\circ} \mathrm{C}$.

\section{Preparación de Muestras}

Las muestras fueron desinfectadas en una solución de hipoclorito de sodio (75 ppm), enjuagadas y drenadas (el agua remanente fue eliminada con papel absorbente). La muestra total se dividió en tres partes iguales (50 g) para cada prueba (muestra control sin escaldar, escaldado en agua y escaldado en solución de $\mathrm{NaCl}$ al 3\%). Además, se determinaron las dimensiones triangulares del equipo de secado, para $50 \mathrm{~g}$ de hojas de quinua se estimó un promedio del área de secado de $0,11 \mathrm{~m}^{2}$, y se determinó el espesor utilizando un micrómetro con precisión $0,01 \mathrm{~mm}$ arrojando un valor de $0,52 \pm 0,1 \mathrm{~mm}$ y semi-espesor de 0,26 $\pm 0,1 \mathrm{~mm}$.

\section{Escaldado}

Los pre-tratamientos de escaldado se realizaron de dos maneras diferentes comúnmente utilizadas para preservar la textura y por otro lado para lograr la desactivación de enzimas en base a estudios previos: Escaldado en agua destilada o solución de cloruro de sodio $(3 \% \mathrm{p} / \mathrm{p})$ a $86^{\circ} \mathrm{C}$ por un periodo de 2 minutos. La relación muestra-solución fue mantenida a 1:20 (p/p). Luego del escaldado, las muestras fueron introducidas en bolsas de polipropileno y enfriadas con agua potable, drenadas y secadas con papel absorbente para la realización de los pre-tratamientos con tres réplicas (Akonor y Tortoe, 2014). Una parte de las hojas de quinua sin escaldar fue guardada como muestra control.

\section{Análisis químico proximal}

El contenido de sólido seco y macronutrientes (proteína, grasa, ceniza, fibra cruda y carbohidratos) en las hojas de quinua con y sin escaldado fue determinado por el método estándar (AOAC, 1990). El análisis fue realizado por triplicado para cada tratamiento.

\section{Equipo de secado}

El secado se realizó en un secador piloto de lecho fijo 0, en la Universidad Nacional del Altiplano-Puno, implementado con control adaptivo Laview (termocuplas en el ingreso de aire a la cámara, centro de cámara, control resistencia eléctrica y velocidad de circulación de aire) de $200 \mathrm{~g}$ de capacidad. Una balanza de precisión RADWAD WTB-2000 POLAND con puerto RS-232 de $0,01 \mathrm{~g}$ de precisión, la cual fue adaptada para el registro continuo de peso a intervalos de $1 \mathrm{~min}$.

\section{Proceso de secado}

Las muestras de hojas de quinua control y las pretratadas fueron secadas a temperaturas de 50, 60 y 70 ${ }^{\circ} \mathrm{C}$. El secador fue calentado previamente por $30 \mathrm{minu}-$ tos operando a condiciones ambientales de $10-20^{\circ} \mathrm{C}$ y humedad relativa (HR) 35-45\%. La corriente de aire fue paralela a la superficie horizontal de las muestras con velocidad de aire constante de $1 \mathrm{~m} \mathrm{~s}^{-1}$. El proceso de secado comenzó cuando las condiciones fueron alcanzadas. Las hojas de quinua fueron extendidas en las bandejas y dispuestas a la cámara de secado comenzando así proceso y el registro de datos que prosiguió hasta alcanzar las condiciones de $\mathrm{X}=0,1 \mathrm{~g} \mathrm{H}_{2} \mathrm{O} \mathrm{g} \mathrm{SS}^{-1}$ ( $\mathrm{SS}=$ sólido seco). Las muestras secas fueron almacenadas en bolsas de polipropileno herméticamente selladas hasta el comienzo de las pruebas de rehidratación.

\section{Determinación del contenido de sólido seco}

El contenido de sólido seco fue determinado por el método estándar (AOAC, 1990). El análisis fue realizado por triplicado para cada tratamiento. El contenido promedio de sólido seco fue calculado usando la siguiente ecuación (1)

$$
X=\frac{W-W_{s}}{W}
$$

Dónde:

$X$ : Humedad expresada en kg totales de agua (kg sólido seco) ${ }^{-1} ; W$ : Peso del sólido húmedo (kg totales de agua); $W_{s}$ : Peso del sólido seco (kg sólido seco). 


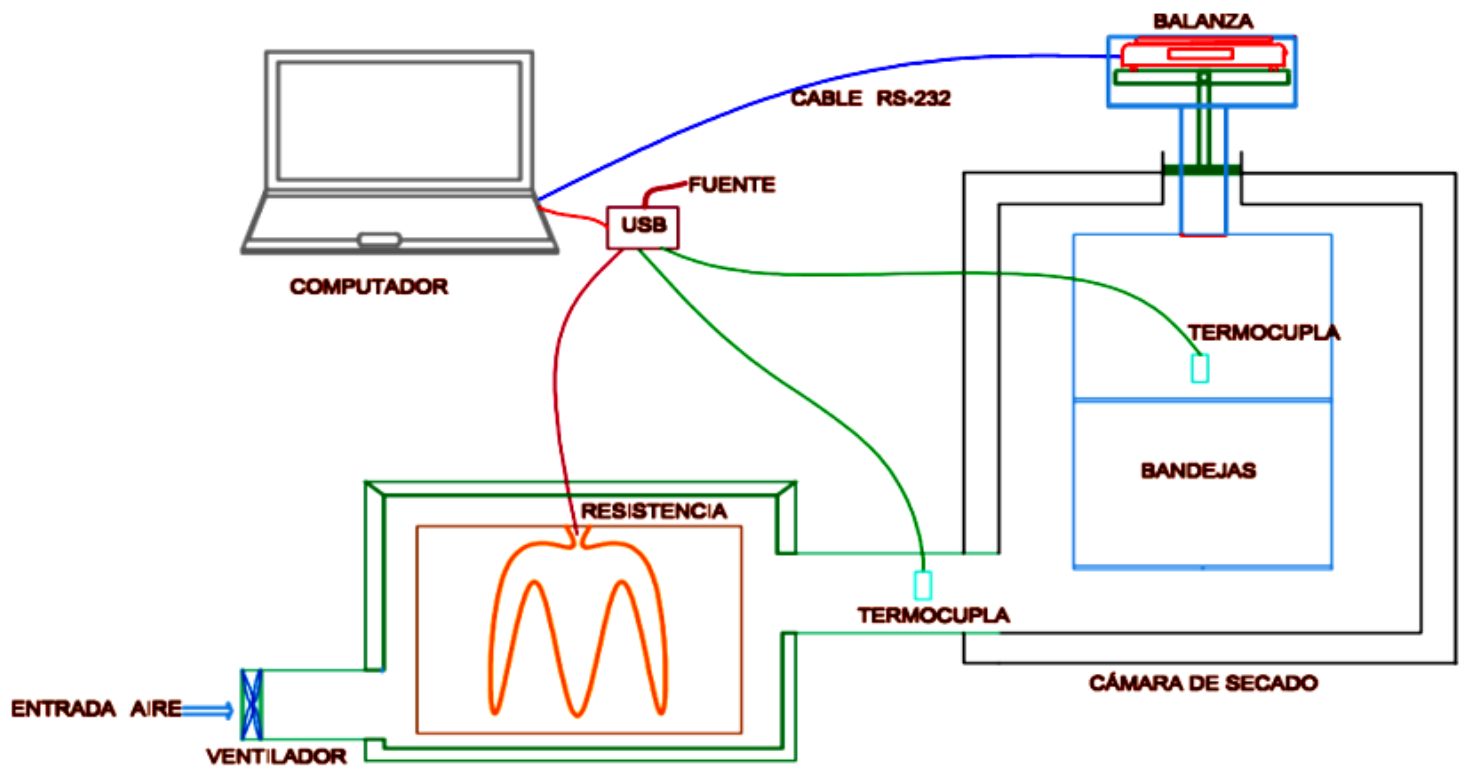

Figura 1. Diagrama del secador y adquisición de datos.

Figure 1. Diagram of the dryer and data acquisition.

\section{Velocidad de secado y ajuste a los modelos matemáticos}

Para calcular las curvas de velocidad de secado, se utilizó un modelo simplificado de la ecuación (2) (Geankoplis, 2006; Ratti, 2009) que se detalla a continuación:

$$
D R=-\frac{L_{s}}{A} \frac{d X}{d t}
$$

Dónde:

$D R$ : velocidad de secado ( $\mathrm{kg} \mathrm{H}_{2} \mathrm{O}$ día $^{-1} \mathrm{~m}^{-2}$ ); $L_{s}$ : $\mathrm{kg}$ sólido seco usado (kg); $A$ : área superficial expuesta al secado $\left(\mathrm{m}^{2}\right)$.

La humedad de la muestra se expresó como el factor adimensional $M R$, ya que existe diferencia de humedad inicial de las muestras, calculado de acuerdo a la ecuación (3):

$$
M R=\frac{M_{t}-M_{e}}{M_{0}-M_{e}}
$$

Debido a que $M_{e}$ es relativamente pequeño en comparación a $M_{t}$ ó $M_{0}$, se asume igual a cero y reduciéndose la expresión a $M R=M_{t} / M_{0}$ (Taheri-Garavand et al., 2011; Akonor y Amankwah, 2012; Darvishi, 2012; Agarry et al., 2013; Motevali et al., 2013; Akonor y Tortoe, 2014; Doymaz, 2014).

Utilizando el programa OrigenPro 2015 los valores experimentales $M R$ se ajustaron a cinco modelos matemáticos de cinéticas de secado: Henderson-Pabis, Midilli, logarítmico, Page y Lewis. Las ecuaciones se detallan en el Cuadro 1.

Para cada modelo se determinó el coeficiente de determinación $\mathrm{R}^{2}$, reducido Chi cuadrado $\chi^{2}$ y la raíz cuadrada de errores cuadráticos RMSE entre los valores experimentales y los valores predichos definidos con las ecuaciones (4), (5) y (6).

$$
R^{2}=\frac{N \sum_{i=1}^{N} M R_{\text {pred }, i} M R_{\text {exp } t, i}-\sum_{i=1}^{N} M R_{\text {pred }, i} \sum_{i=1}^{N} M R_{\exp t, i}}{\sqrt{\left(N \sum_{i=1}^{N} M R_{\text {pred }, i}^{2}-\left(\sum_{i=1}^{N} M R_{\text {pred }, i}\right)^{2}\right)}\left(N \sum_{i=1}^{N} M R_{\exp t, i}^{2}-\left(\sum_{i=1}^{N} M R_{\exp t, i}\right)^{2}\right)}
$$


Cuadro 1. Modelos matemáticos referidos para las curvas de secado.

Table 1. Mathematical models referred to for drying curves.

\begin{tabular}{|c|c|c|c|}
\hline $\mathbf{N}^{\circ}$ & Nombre del modelo & Modelo & Referencias \\
\hline 1 & Henderson y Pabis & $M R=\frac{M_{t}-M_{e}}{M_{0}-M_{e}}=a \exp (-k t)$ & Doymaz (2014), Simha y Gugalia (2013) \\
\hline 2 & Midilli & $M R=\frac{M_{t}-M_{e}}{M_{0}-M_{e}}=a \exp \left(-k t^{n}\right)+b t$ & $\begin{array}{l}\text { Midilli et al. (2002), Ratti (2009) y Simha y } \\
\text { Gugalia (2013) }\end{array}$ \\
\hline 3 & Logarítmico & $M R=\frac{M_{t}-M_{e}}{M_{0}-M_{e}}=a \exp (-k t)+c$ & Doymaz (2014), Ratti (2009) \\
\hline 4 & Page & $M R=\frac{M_{t}-M_{e}}{M_{0}-M_{e}}=\exp \left(-k t^{n}\right)$ & Doymaz (2014) \\
\hline 5 & Lewis & $M R=\frac{M_{t}-M_{e}}{M_{0}-M_{e}}=\exp (-k t)$ & Doymaz (2014), Ratti (2009) \\
\hline
\end{tabular}

$$
\begin{gathered}
x^{2}=\frac{\sum_{i=1}^{N}\left(M R_{\text {exp }, i}-M R_{\text {pred }, i}\right)}{N-Z} \\
R M S E=\sqrt{\frac{\sum_{i=1}^{N}\left(M R_{\text {exp }, i}-M R_{\text {pred }, i}\right)^{2}}{N}}
\end{gathered}
$$

El modelo que mejor describe la cinética de secado fue elegido como el modelo con el menor valor $\chi^{2}$ y RMSE y con el mayor valor $R^{2}$ (Meisami-asl y Rafiee, 2009; Abano et al., 2011; Darvishi, 2012; Akonor y Tortoe, 2014; Doymaz, 2014).

\section{Difusividad efectiva del agua y energía de activación}

Generalmente, la difusión es asumida como el mecanismo de transporte dominante durante el secado y la velocidad del movimiento de humedad es por lo tanto descrito por el valor de la $D_{\text {eff }}$ la cual está relacionada con $M R$ por la ecuación (7) para lámina infinita (Crank, 1975; Akonor y Tortoe, 2014; Doymaz, 2014):

$$
M R=\frac{M_{t}-M_{e}}{M_{o}-M_{e}}=\frac{8}{\pi^{2}} \sum_{n=0}^{\infty} \frac{1}{(2 n+1)} \exp \left[-(2 n+1)^{2} \pi^{2} D_{\text {eff }} \frac{t}{4 L^{2}}\right]
$$

La misma que puede convertirse a ecuación lineal:

$$
\ln (M R)=\ln \left(\frac{8}{\pi^{2}}\right)-K * t \quad \text { Donde } K=\frac{\pi^{2} D_{e f f}}{4 L^{2}}
$$




\section{Rehidratación}

Se evaluó la rehidratación de las muestras secadas, para lo cual se pesó $1 \pm 0,03 \mathrm{~g}$ de muestra seca y se rehidrató remojándolos en vasos de precipitado de $250 \mathrm{ml}$ con agua destilada (muestra:agua - $1: 100$ ) a $50 \pm 1{ }^{\circ} \mathrm{C}$ (Doymaz, 2014). Las muestras fueron removidas a intervalos de 30 minutos, el exceso de agua fue eliminada cuidadosamente con papel absorbente, se pesó en una balanza electrónica (METLER TOLEDO monoblock PB3002-S, Switzerland) e inmediatamente devuelto al agua de remojo (Akonor y Tortoe, 2014). La prueba se llevó por 4 horas y la relación de rehidratación fue calculada con la ecuación (11).

$$
R R=\frac{W_{r}}{W_{d}}
$$

Dónde:

$R R$ : Razón de rehidratación; $W_{r}$ : Peso total al final del proceso de rehidratación $(\mathrm{kg}) ; W_{d}$ : Peso de la materia seca $(\mathrm{kg})$.

\section{Análisis estadístico}

Para el análisis estadístico de los datos se utilizó OrigenPro 2015. El efecto del escaldado en la composición química proximal, así como de la temperatura, tiempo de secado, difusidad efectiva y relación de rehidratación fueron evaluados mediante ANOVA con comparación múltiple de Tukey, con el fin de determinar diferencias significativas de los datos al $\mathrm{P}<0,05$. Los datos fueron expresados como media \pm desviación estándar para los datos en base a tres repeticiones por muestra y análisis.

Los parámetros de los modelos matemáticos fueron calculados a través de regresión no lineal con proceso iterativo del algoritmo de Levenberg-Marquardt.

\section{RESULTADOS Y DISCUSIÓN}

\section{Análisis químico proximal}

Los resultados de la composición química proximal de las hojas de quinua muestras control y sometidas a escaldado, en términos de sólido seco se presenta en el Cuadro 2 con sus respectivas desviaciones estándar con letras diferentes indicando diferencias estadísticamente significativas al 95\% de confianza. El contenido proteico en las muestras M1 (28,37\%) y M2 (29,88\%) fueron inferiores a M0 $(30,47 \%)$, este resultado se atribuye a la hidrólisis durante el escaldado con pérdida de proteínas hidrosolubles y/o compuestos nitrogenados no proteicos. Así como es sostenido por previas investigaciones (Jongen, 2002; Reyes et al., 2004) que siendo el escaldado un proceso de tratamiento térmico conlleva a inevitables perdidas de compuestos hidrosolubles especialmente en el escaldado en agua. Pero contradiciendo con Ayorkor (2009) que reportó diferencia no significativa del contenido proteico para muestras frescas y escaldadas de Moringa oleífera Lam. y Moringa stenopetala (Baker f.) atribuyendo la misma a la diferencia de especie.

El contenido graso en las muestras escaldada en agua (M1) $(8,13 \%)$ fueron inferiores que las muestras escaldadas con $\mathrm{NaCl}$ (M2) $(8,47 \%)$, pero no presentan diferencias significativas con respecto a la muestra control M0 (8,49\%) estos resultado se deben a la poca solubilidad de la grasa en el proceso de escaldado. Acorde con estudios previos (Ayorkor, 2009; Jongen, 2002; Reyes et al., 2004; Veda, 2009) donde señalan que con el escaldado los compuestos lipídicos son mejor retenidas y/o fijadas especialmente el $\beta$-caroteno y la clorofila que son termo-resistentes. Mientras Schlick (2000) indica que las hojas de quinua contienen adecuados porcentajes de los ácidos grados esenciales, como el ácido linoleico, para la dieta humana $(13,6 \%$

Cuadro 2. Resultados del análisis químico proximal de hojas de quinua sin escaldar y escaldados expresados en porcentajes de base seca.

Table 2. Results of the proximal chemical analysis of quinoa leaves without scalding and scalding expressed in percentajes of dry base.

\begin{tabular}{lccc}
\hline \multicolumn{1}{c}{ COMPONENTES } & CONTROL (M0) & ESCALDADO EN AGUA (M1) & ESCALDADO EN NaCl (M2) \\
\hline Proteína & $30,47 \pm 0,26 \mathrm{a}$ & $28,37 \pm 0,42 \mathrm{~b}$ & $29,88 \pm 0,11 \mathrm{a}$ \\
Grasa & $8,49 \pm 0,12 \mathrm{a}$ & $8,13 \pm 0,31 \mathrm{a}$ & $8,47 \pm 0,28 \mathrm{a}$ \\
Ceniza & $20,03 \pm 0,27 \mathrm{a}$ & $17,17 \pm 0,42 \mathrm{~b}$ & $20,95 \pm 0,35 \mathrm{a}$ \\
Hidratos de carbono & $28,00 \pm 0,61 \mathrm{a}$ & $36,27 \pm 0,51 \mathrm{~b}$ & $27,85 \pm 0,29 \mathrm{a}$ \\
Fibra & $13,00 \pm 0,18 \mathrm{a}$ & $10,07 \pm 0,02 \mathrm{~b}$ & $12,85 \pm 0,23 \mathrm{a}$ \\
\hline
\end{tabular}

Las medias seguidas por la misma letra no son significativamente diferentes $(\mathrm{P}<0,05)$ según el análisis de varianza y la comparación múltiple de Tukey. 
de la fracción lipídica). Dicho ácido graso es importante en la formación muscular y el transporte, ruptura y excreción del colesterol. Por lo que, el alto contenido graso en las hojas de esta variedad podría ser aprovechada de manera funcional.

El contenido de cenizas en M1 $(17,17 \%)$ fue inferior que en M0 (20,03\%), este resultado se atribuye a la pérdida de minerales durante el proceso de escaldado, donde las sales minerales quedan en el agua del escaldado. Un caso contrario fue detectado en M2, ya que esta última presenta los iones sodio y cloro que son absorbidos por la muestra hasta el equilibrio durante el proceso de escaldado. Fundamentándose en estudios de Mepba et al. (2007) quienes indican que tanto el escaldado y la cocción causan reducciones de minerales significativas (K, Na, Ca, Zn, Fe y P) en amaranto, espinaca y otros vegetales. De estos minerales $\mathrm{K}, \mathrm{Ca}$, P y Mg fueron reportados en mayores concentraciones en las hojas de quinua por Galarza (2010) y Schlick (2000), este último indica que la variación en el contenido de minerales se atribuye a las diferentes composiciones del suelo. Por otro lado, Ayorkor (2009) indica que el contenido de ceniza fue menor en las hojas escaldadas en relación a las no escaldadas en M. Oleifera como $M$. stenopetala atribuyendo dicha variación a la solubilidad de estos micro-elementos en el escaldado.

El contenido de fibra en las muestras M1 $(10,07 \%)$ fueron inferiores que M0 (13,00\%) y M2 (12,85\%), estos resultados se pueden atribuir a la perdida de fibra soluble durante el escaldado en agua, mientras que en solución de $\mathrm{NaCl}$ el contenido de fibra es fijado. Estos resultados se fundamentan en estudios de Ayorkor (2009) y Mepba et al. (2007) quienes indican que tanto el escaldado como la cocción causan significativa variación de la fibra cruda en amaranto, moringa, espinaca y otros vegetales. En los estudios realizados por Schlick (2000) en las hojas de quinua indica que el contenido de fibra aumenta cuando existe alto estrés medio ambiental al que se exponen los cultivos. Por otro lado Galarza (2010) y Ayorkor (2009) mencionan que el contenido de fibra en los alimentos es importante en el flujo gastrointestinal por lo que el contenido en fibra de las hojas de quinua presenta gran potencial.

El contenido de hidratos de carbono en M2 $(27,85 \%)$ fue inferior que en M1 (36,27\%), este resul- tado se atribuye a la pérdida de hidratos de carbono en el medio de escaldado, en el caso de M2 los azúcares de las hojas de quinua difunden con más facilidad en la solución de $\mathrm{NaCl}$ que en agua. El contenido superior de hidratos de carbono en M1 se explica con el hecho de que al realizarse el proceso de escaldado en agua los demás componentes se reducen, considerando a la vez que la humedad en base húmeda de M1 fue superior a M2 y por ende a M0. Al relacionar estos resultados con productos similares en la literatura (Mepba et al., 2007; Ayorkor, 2009) indican que los efectos del proceso sobre el contenido de hidratos de carbono son mínimos pero igualmente encontraron diferencia entre especies.

\section{Humedad inicial}

El Cuadro 3 presenta la humedad inicial de las hojas de quinua. En las muestras sin pre-tratamiento M0 utilizadas en la presente investigación fue de 82,69 \pm $0,17 \%$ en base húmeda ó $4,77 \mathrm{~g} \mathrm{H}_{2} \mathrm{O} \mathrm{g} \mathrm{SS}^{-1}$. M1 presentó $86,63 \pm 0,05 \%$ ó $6,47 \mathrm{~g} \mathrm{H}_{2} \mathrm{O} \mathrm{g} \mathrm{SS}^{-1}$; mientras que M2 presentó $78,12 \pm 0,11 \%$ ó $3,57 \mathrm{~g} \mathrm{H}_{2} \mathrm{O} \mathrm{g} \mathrm{SS}{ }^{-1}$. Estos resultados son superiores a los valores reportados por Kozioł (1992), Schlick (2000) y Galarza (2010) para hojas frescas de quinua, equivalente a $77,80 \pm 0,33 \%$ ó $3,50 \mathrm{~g}$ $\mathrm{H}_{2} \mathrm{O} \mathrm{g} \mathrm{SS}^{-1}$. Estos resultados se atribuyen a la baja humedad relativa del Altiplano y como consecuencia de esto, los cultivos tienden a retener mayor cantidad de agua en sus tejidos.

En la Figura 2 se presenta la variación de la humedad en función del tiempo de secado hasta alcanzar X

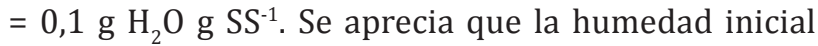
de M0 varía como consecuencia de las condiciones ambientales y del proceso de desinfección del cual no se eliminó el agua remanente. En M1 se apreció un incremento de la humedad inicial como consecuencia de absorción de agua por las células durante el pre-tratamiento y la pérdida de material hidrosoluble. Para el caso de M2, la humedad inicial disminuye cerca del 19,48\% del contenido de agua libre en comparación con M1 y M0. Estos resultados son similares para procesos de secado de alimentos sometidos a sistemas de secado combinados (Fellows, 2000; Severini et al., 2005; Akonor y Tortoe, 2014).

Cuadro 3. Resultados de la humedad inicial de las muestras.

Table 3. Results of the inicial humidity of the samples.

\begin{tabular}{|c|c|c|c|}
\hline Muestra & Humedad (\% b.h.) & $X\left(\mathrm{~kg} \mathrm{H}_{2} \mathrm{O} \mathrm{kg} \mathrm{SS} S^{-1}\right)$ & 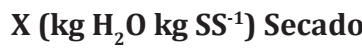 \\
\hline M0 & $82,69 \pm 0,17$ & 4,77 & 4,78 \\
\hline M1 & $86,63 \pm 0,05$ & 6,47 & 6,47 \\
\hline M2 & $78,12 \pm 0,11$ & 3,57 & 3,57 \\
\hline
\end{tabular}




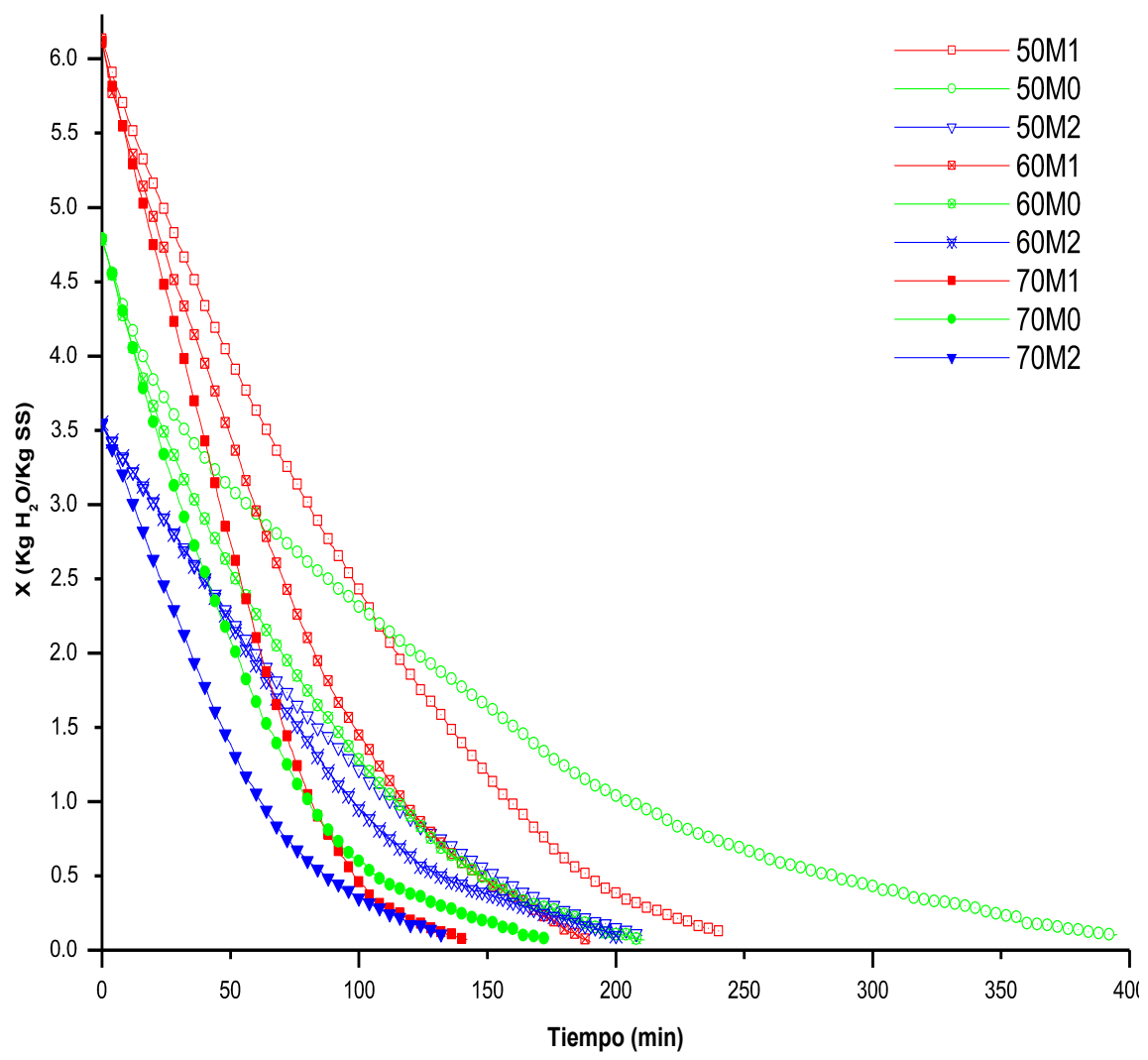

Figura 2. Pérdida de humedad en función del tiempo.

Figure 2. Loss of humidity as a function of time.

\section{Tiempo de secado hasta el equilibrio}

La Figura 3 presenta los resultados de la variación del tiempo de secado experimental hasta un nivel de

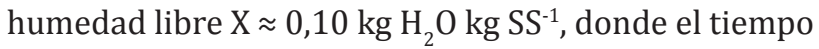
está expresado como la media \pm SD seguido de letras diferentes en superíndice indicando diferencia significativa al 95\% de confianza. Es evidente la dependencia del tiempo de secado con respecto al pre-tratamiento y a la temperatura aplicada. Queda demostrado que mientras mayores sean las temperaturas y cuando se pre-traten las muestras previo al secado, menores resultan ser los tiempos requeridos para el secado de las hojas de quinua. Lo cual concuerda directamente con investigaciones previas realizadas a diferentes productos alimenticios (Severini et al., 2005; Silva et al., 2008; Aghbashlo et al., 2009; Jokić et al., 2009; Abano et al., 2011; Espinoza, 2011; Da Rocha et al., 2012; Doymaz, 2012; Upadhyaya et al., 2012; Agarry et al., 2013; Doymaz, 2014; Sonmete et al., 2017).

\section{Velocidad de secado}

En la Figura 4 se representa la curva de velocidad de secado (DR) en función del tiempo (t). Se observa que en los primeros 100 minutos del proceso la evaporación de agua es alta en las muestras escaldadas bajo los dos sistemas en comparación de la muestra control. Posteriormente, disminuyen lentamente a medida que la humedad de la matriz de las muestras es liberada con más dificultad. Estos resultados concuerdan con estudios previos en diferentes productos alimentarios (Severini et al., 2005; Abano et al., 2011; Da Rocha et al., 2012; Doymaz, 2012; Upadhyaya et al., 2012; Agarry et al., 2013; Doymaz, 2014).

La Figura 5 muestra que la velocidad de periodo decreciente predominó tanto en las muestras escaldadas como en la muestra control durante el secado a diferentes temperaturas. Ello significa que el mecanismo físico que genera el movimiento de la humedad es la difusión del vapor de agua o agua ligada a través del tejido seco hacia el aire seco en velocidades cada vez más lentas en comparación a la velocidad de evaporación desde la superficie (Doymaz, 2014).

Con el escaldado se provocaron cambios en la estructura celular y consigo un aumento de la velocidad de secado. Además, los solutos del $\mathrm{NaCl}$ incrementaron la viscosidad, provocando así, la disminución de la actividad de agua, reduciendo la velocidad del movimiento del agua como indica Fellows (2000). Aunque no fue determinado 


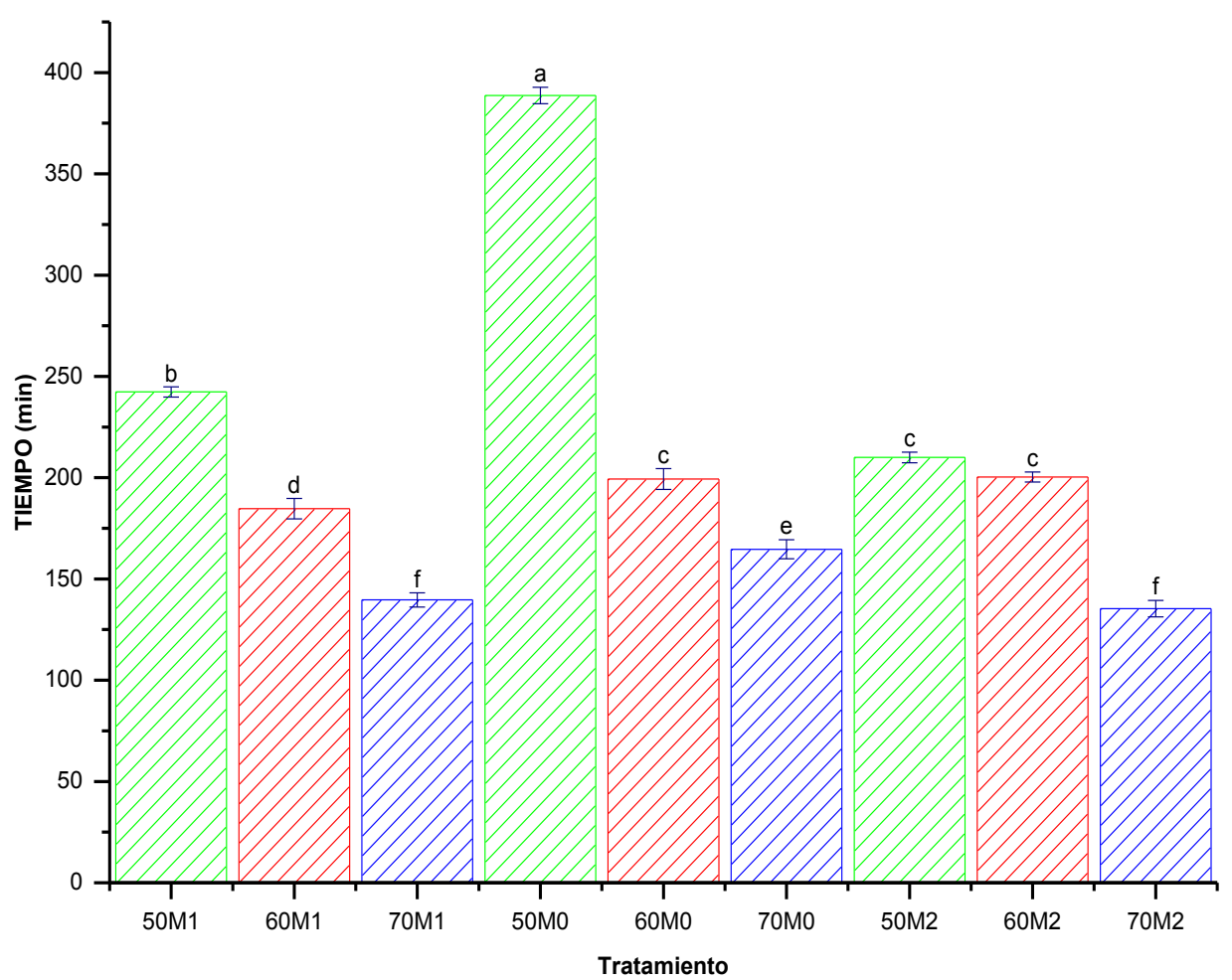

Figura 3. Tiempo de secado hasta alcanzar $\mathrm{X}=0,1 \mathrm{~kg} \mathrm{H}_{2} \mathrm{O} \mathrm{kg} \mathrm{SS}-1$ ( $\mathrm{SS}=$ sólido seco). Letras diferentes representan que existe diferencia significativa al nivel de $\mathrm{P}<0,05$.

Figure 3. Drying time until reaching $\mathrm{X}=0.1 \mathrm{~kg} \mathrm{H}_{2} \mathrm{O} \mathrm{kg} \mathrm{DS}^{-1}$ (DS = dry solid). Different letters represent that there is a significant difference at the level of $\mathrm{P}<0.05$.

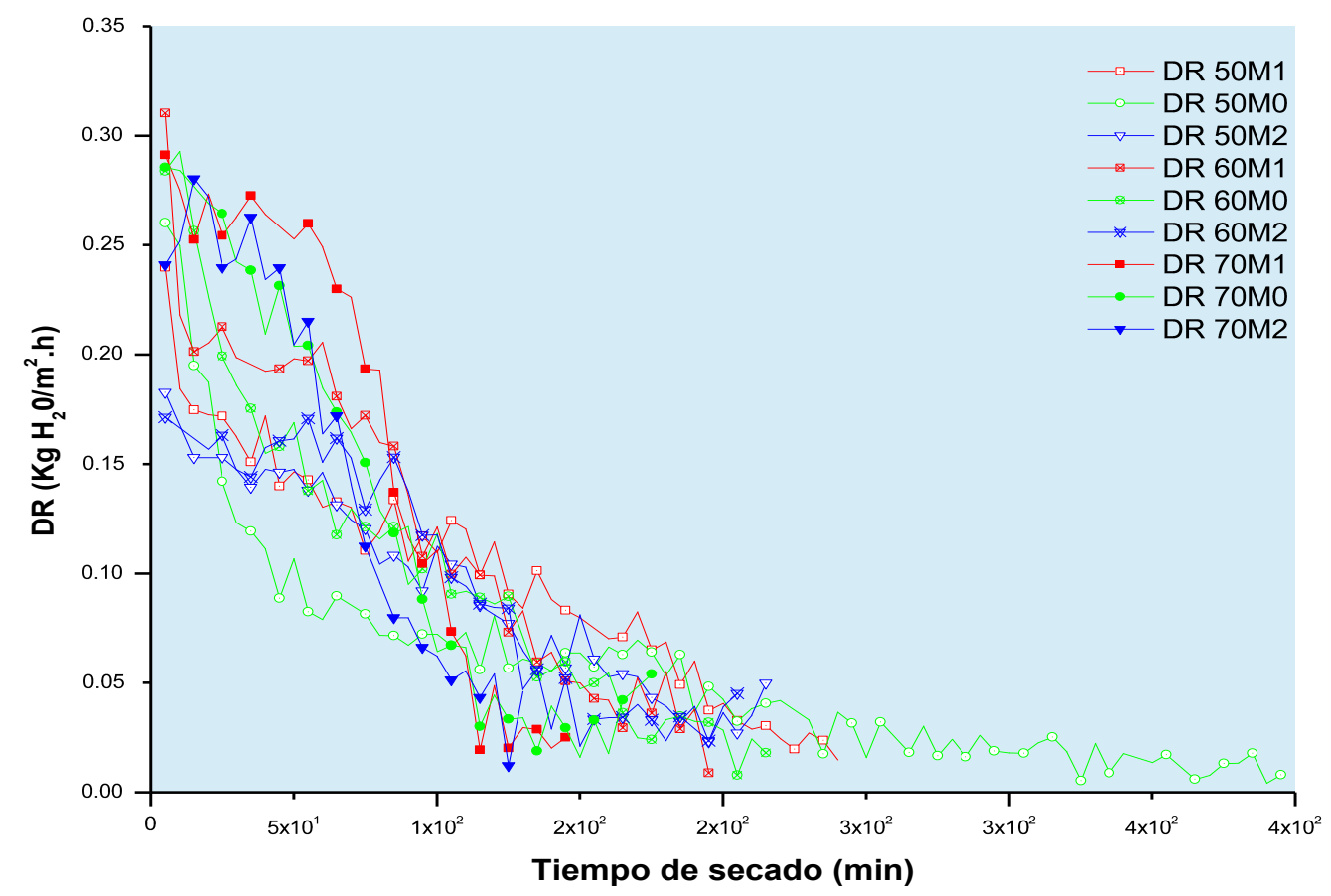

Figura 4. Velocidad de secado en función del tiempo.

Figure 4. Drying speed as a function of time. 


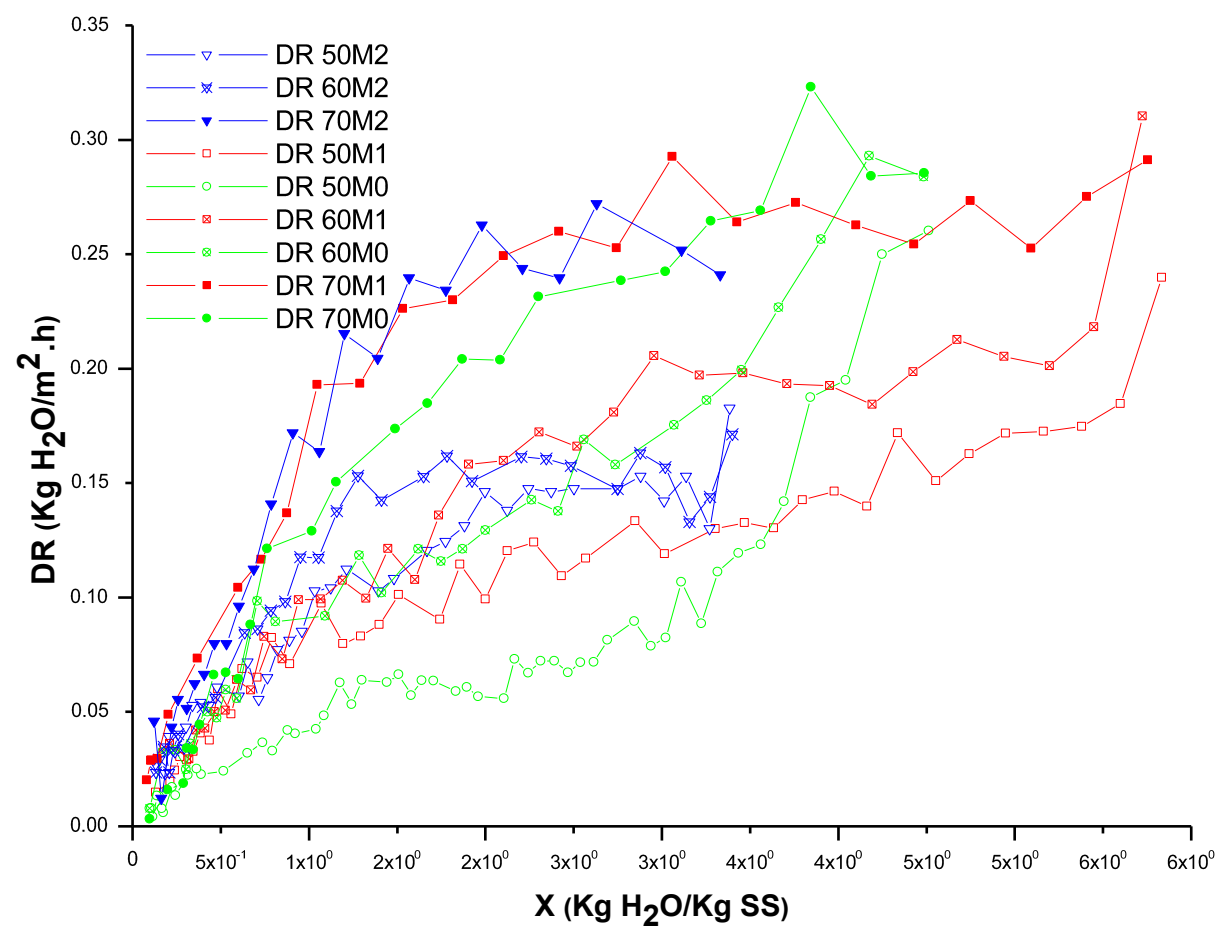

Figura 5. Velocidad de secado en función del contenido de humedad.

Figure 5. Drying speed as a function of humidity content.

con exactitud el punto de la humedad crítica debido al encogimiento que ocurre durante el secado, varía de 3,5 a 5,0 kg agua $\mathrm{kg} \mathrm{SS}^{-1}$ para vegetales Rao et al. (2005). Las curvas de velocidad de secado para M0 son similares que para espinaca y alfalfa (Darvishi, 2011; Prasad y Prasad, 2013). Las curvas para M1 y M2 concuerdan con Jokić et al. (2009) quienes encontraron que muestras pre-tratadas de papa presentaron velocidades de secado mayores que las muestran sin pre-tratar, asimismo Doymaz (2014) detectó que tanto las muestras pre-tratadas y sin pre-tratar de champiñones presentan ambos periodos de secado. En la aplicación de altas temperaturas se reduce el periodo de velocidad constante, comportamiento similar reportó Abano et al. (2011) en el secado de rodajas de tomate indicando que para mayor temperatura no se observó el periodo a velocidad constante.

\section{Modelado}

La relación de humedad $(M R)$ en función del tiempo de secado experimental fue ajustada a los modelos disponibles en la literatura del Cuadro 1.

Los resultados obtenidos para los modelos realizados en base a los datos experimentales se presentan en el Cuadro 4, donde se muestran los valores estimados de las constantes con sus correspondientes valores estadísticos $R^{2}, \chi^{2}$ y RMSE para cada tratamiento. Los valores variaron de 0,99954 a 0,$9539 ; 0,000040$ a $4,57^{-3}$ y de 0,005330 a 0,6986 ; respectivamente. Los modelos de Page y Midilli resultaron tener mejor ajuste y describen la cinética de secado de las hojas de quinua al ser significativamente diferentes del resto de modelos $\mathrm{P}<0,05$. Así los valores de $R^{2}, \chi^{2}$, y RMSE para el modelo de Page variaron de 0,992390 a 0,99954; 0,000040 a 0,000507 y 0,005330 a 0,404650 ; respectivamente. Por otro lado, para el modelo de Midilli variaron de 0,9925 a 0,$9985 ; 0,000123$ a 0,000488 y 0,0167 a 0,3987; respectivamente.

La Figura 6 muestra los valores predichos por los dos modelos en función de los valores experimentales donde se aprecia que los valores estimados se encuentran alrededor de la línea diagonal de $45^{\circ}$. Estos resultados concuerdan con varios autores que han estudiado el modelado del secado de alimentos (Silva et al., 2008; Abano et al., 2011; Taheri-Garavand et al., 2011; Akonor y Amankwah, 2012; Da Rocha et al., 2012; Upadhyaya et al., 2012; Darvishi et al., 2013) quienes señalan que los modelos de Page como Midilli presentan un buen ajuste. Aunque depende principalmente del producto sometido al proceso de secado, así como de las condiciones (entero, laminado, etc.) y además de las condiciones propias establecidas para el proceso (temperaturas, pre-tratamiento y velocidad del aire). Por otro lado, se observa que el valor de la constante " $k$ " $\left(\mathrm{min}^{-1}\right)$ presente en los modelos de Midilli, logarítmico, Page, Lewis y Henderson-Pabis tienden a aumentar si 
Cuadro 4. Resultados de constantes y análisis estadísticos para los modelos estudiados.

Table 4. Results of constants and statistical analyzes for the models studied.

\begin{tabular}{|c|c|c|c|c|c|c|c|c|c|}
\hline \multirow{2}{*}{ MODELO } & \multirow[t]{2}{*}{ TRAT } & \multicolumn{5}{|c|}{ CONSTANTES } & \multicolumn{3}{|c|}{ ESTADISTICO } \\
\hline & & $\mathbf{a}$ & $\mathbf{k}$ & n & $\mathbf{b}$ & C & $\chi^{2}$ & $\mathbf{R}^{2}$ & RMSE \\
\hline \multirow{9}{*}{ : } & $50 \mathrm{M} 1$ & 1,0000 & 0,00367 & 1,1777 & $-3,8 \mathrm{E}-04$ & & $2,92 \mathrm{E}-04$ & 0,9965 & 0,0697 \\
\hline & $50 \mathrm{M} 0$ & 0,9990 & 0,00769 & 1,0000 & $1,0 \mathrm{E}-06$ & & $4,88 \mathrm{E}-04$ & 0,9925 & 0,3987 \\
\hline & $50 \mathrm{M} 2$ & 1,0000 & 0,00233 & 1,3400 & $-1,0 \mathrm{E}-10$ & & $2,32 \mathrm{E}-04$ & 0,9973 & 0,0508 \\
\hline & $60 \mathrm{M} 1$ & 1,0000 & 0,00317 & 1,3420 & $-9,0 \mathrm{E}-05$ & & $3,07 \mathrm{E}-04$ & 0,9965 & 0,0586 \\
\hline & $60 \mathrm{M0}$ & 1,0000 & 0,00937 & 1,0529 & $-3,1 \mathrm{E}-04$ & & $1,75 \mathrm{E}-04$ & 0,9977 & 0,0771 \\
\hline & $60 \mathrm{M} 2$ & 0,9988 & 0,0015 & 1,4635 & $9,2 \mathrm{E}-06$ & & $2,44 \mathrm{E}-04$ & 0,9973 & 0,0515 \\
\hline & $70 \mathrm{M} 1$ & 1,0000 & 0,0020 & 1,5341 & $-0,0001$ & & $4,21 \mathrm{E}-04$ & 0,9958 & 0,0635 \\
\hline & $70 \mathrm{M} 0$ & 1,0000 & 0,00603 & 1,2594 & $-9,3 E-06$ & & $1,23 \mathrm{E}-04$ & 0,9985 & 0,0452 \\
\hline & $70 \mathrm{M} 2$ & 0,9970 & 0,00493 & 1,3397 & $1,0 \mathrm{E}-04$ & & $1,28 \mathrm{E}-04$ & 0,9985 & 0,0167 \\
\hline \multirow{9}{*}{ 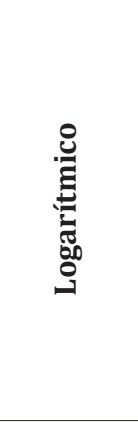 } & $50 \mathrm{M} 1$ & 1,0010 & 0,00103 & & & $-0,0001$ & $2,09 \mathrm{E}-03$ & 0,9750 & 0,5007 \\
\hline & $50 \mathrm{M} 0$ & 1,0465 & 0,00700 & & & $-0,0450$ & 4,34E-04 & 0,9933 & 0,3548 \\
\hline & $50 \mathrm{M} 2$ & 0,9988 & 0,01170 & & & 0,0070 & 2,31E-03 & 0,9732 & 0,5073 \\
\hline & $60 \mathrm{M} 1$ & 1,0640 & 0,01329 & & & $-0,0639$ & $9,42 \mathrm{E}-04$ & 0,9893 & 0,1809 \\
\hline & $60 \mathrm{M} 0$ & 1,1100 & 0,01071 & & & $-0,1107$ & $9,08 \mathrm{E}-05$ & 0,9988 & 0,0401 \\
\hline & $60 \mathrm{M} 2$ & 1,0050 & 0,01213 & & & $-0,0111$ & $2,90 \mathrm{E}-03$ & 0,9685 & 0,6145 \\
\hline & $70 \mathrm{M} 1$ & 1,0750 & 0,01650 & & & $-0,0722$ & $2,54 \mathrm{E}-03$ & 0,9744 & 0,3858 \\
\hline & $70 \mathrm{M} 0$ & 1,0100 & 0,01705 & & & $-0,0252$ & 8,73E-04 & 0,9894 & 0,3212 \\
\hline & $70 \mathrm{M} 2$ & 1,0020 & 0,01978 & & & $-0,0010$ & $1,88 \mathrm{E}-03$ & 0,9785 & 0,2476 \\
\hline \multirow{9}{*}{ 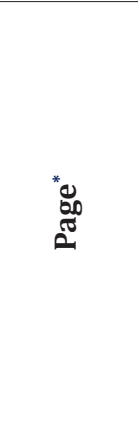 } & $50 \mathrm{M} 1$ & & 0,00299 & 1,2624 & & & $5,07 \mathrm{E}-04$ & 0,9939 & 0,1221 \\
\hline & $50 \mathrm{M0}$ & & 0,00842 & 0,9850 & & & $4,94 \mathrm{E}-04$ & 0,9924 & 0,4047 \\
\hline & $50 \mathrm{M} 2$ & & 0,00280 & 1,3002 & & & $2,08 \mathrm{E}-04$ & 0,9976 & 0,0460 \\
\hline & $60 \mathrm{M} 1$ & & 0,00406 & 1,3015 & & & $1,62 \mathrm{E}-04$ & 0,9982 & 0,0312 \\
\hline & $60 \mathrm{M} 0$ & & 0,00679 & 1,1600 & & & $4,28 \mathrm{E}-04$ & 0,9944 & 0,1896 \\
\hline & $60 \mathrm{M} 2$ & & 0,00220 & 1,3841 & & & $1,18 \mathrm{E}-04$ & 0,9987 & 0,0252 \\
\hline & $70 \mathrm{M} 1$ & & 0,00259 & 1,4817 & & & $3,01 \mathrm{E}-04$ & 0,9970 & 0,0461 \\
\hline & $70 \mathrm{M} 0$ & & 0,00723 & 1,2221 & & & 6,57E-05 & 0,9992 & 0,0243 \\
\hline & $70 \mathrm{M} 2$ & & 0,00598 & 1,2953 & & & 4,01E-05 & 0,9995 & 0,0053 \\
\hline \multirow{9}{*}{ 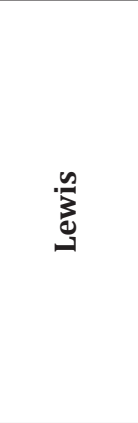 } & $50 \mathrm{M} 1$ & & 0,01028 & & & & $2,08 \mathrm{E}-03$ & 0,9751 & 0,5042 \\
\hline & $50 \mathrm{M} 0$ & & 0,00795 & & & & $5,55 \mathrm{E}-04$ & 0,9915 & 0,4548 \\
\hline & $50 \mathrm{M} 2$ & & 0,01140 & & & & $2,21 \mathrm{E}-03$ & 0,9744 & 0,4901 \\
\hline & $60 \mathrm{M} 1$ & & 0,01511 & & & & $2,04 \mathrm{E}-03$ & 0,9768 & 0,3949 \\
\hline & $60 \mathrm{M0}$ & & 0,01365 & & & & $8,10 \mathrm{E}-04$ & 0,9894 & 0,3597 \\
\hline & $60 \mathrm{M} 2$ & & 0,01247 & & & & $3,00 \mathrm{E}-03$ & 0,9674 & 0,6412 \\
\hline & $70 \mathrm{M} 1$ & & 0,01891 & & & & 4,39E-03 & 0,9557 & 0,6760 \\
\hline & $70 \mathrm{M} 0$ & & 0,01832 & & & & $1,08 \mathrm{E}-03$ & 0,9869 & 0,3986 \\
\hline & $70 \mathrm{M} 2$ & & 0,01975 & & & & $1,88 \mathrm{E}-03$ & 0,9784 & 0,2525 \\
\hline \multirow{9}{*}{ 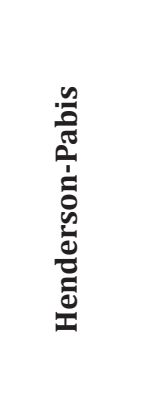 } & $50 \mathrm{M} 1$ & 1,0000 & 0,01032 & & & & $2,02 \mathrm{E}-03$ & 0,9757 & 0,5045 \\
\hline & $50 \mathrm{M} 0$ & 0,9990 & 0,00762 & & & & $4,93 \mathrm{E}-04$ & 0,9924 & 0,4039 \\
\hline & $50 \mathrm{M} 2$ & 1,0000 & 0,01123 & & & & $2,19 \mathrm{E}-03$ & 0,9746 & 0,4831 \\
\hline & $60 \mathrm{M} 1$ & 1,0010 & 0,01556 & & & & $2,10 \mathrm{E}-03$ & 0,9761 & 0,4056 \\
\hline & $60 \mathrm{M0}$ & 1,0050 & 0,01420 & & & & 8,83E-04 & 0,9885 & 0,3913 \\
\hline & $60 \mathrm{M} 2$ & 1,0000 & 0,01294 & & & & $3,14 \mathrm{E}-03$ & 0,9659 & 0,6684 \\
\hline & $70 \mathrm{M} 1$ & 1,0000 & 0,01972 & & & & $4,57 \mathrm{E}-03$ & 0,9539 & 0,6986 \\
\hline & $70 \mathrm{M} 0$ & 1,0000 & 0,01878 & & & & 1,13E-03 & 0,9862 & 0,4164 \\
\hline & $70 \mathrm{M} 2$ & 1,0000 & 0,02039 & & & & 1,99E-03 & 0,9772 & 0,2649 \\
\hline
\end{tabular}




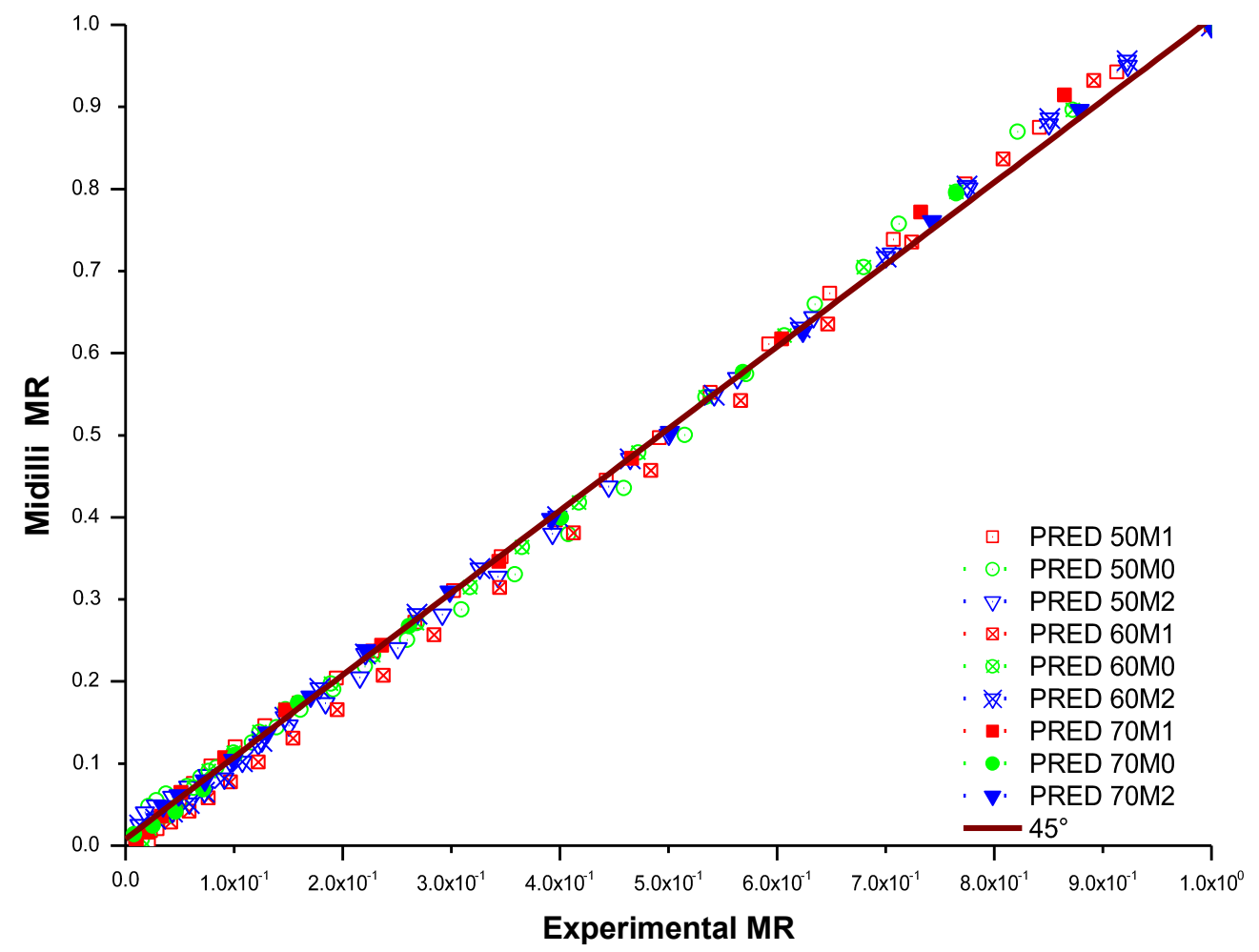

Figura 6. Comparación de relación de humedad entre valores experimentales y predichos con modelos de Page y Midilli.

Figure 6. Comparison of humidity relationship between experimental and predicted values with Page and Midilli models.

las muestras son pre-tratadas y a medida que aumenta la temperatura de secado. Este resultado concuerda con lo obtenido por varios autores (Aghbashlo et al., 2009; Espinoza, 2011; Da Rocha et al., 2012; Olurin et al., 2012) donde se comprueba que esta constante cinética es dependiente de la temperatura y muestra una tendencia a aumentar con el pre-tratamiento, pero las constantes "n", "a", y "b" podrían estar ligadas a la estructura del tejido del alimento y a la velocidad del aire de secado, más que a la temperatura.

\section{Difusividad efectiva del agua}

Los resultados obtenidos de $D_{\text {eff }}$ se presentan en el Cuadro 5, cuyos valores varían entre $3,871 \times 10^{-12} \mathrm{y}$ $1,175 \times 10^{-11}$ denotando un claro aumento al aumentar la temperatura y con los pre-tratamientos, lo que se encuentran rangos normales esperados de $10^{-12}$ a $10^{-8}$ $\mathrm{m}^{2} \mathrm{~s}^{-1}$ para materiales alimentarios (Abano et al., 2011; Darvishi, 2012; Doymaz, 2014). Los resultados mostraron diferencias significativas según ANOVA para el mismo pre-tratamiento, en base a las diferentes temperaturas aplicadas $(\mathrm{P}<0,05)$. Cabe destacar que existe un claro aumento de la difusividad efectiva del agua en las muestras pre-tratadas y al aumentar la temperatura del aire de secado para un determinado pre-tratamien- to. Se efectuó el análisis de comparaciones múltiples de Tukey y se determinó que no existen diferencias estadísticamente significativas entre los tratamientos con la aplicación de $60^{\circ} \mathrm{C}$.

Un caso similar fue reportado en espinacas sin tratamiento de 2,150 a 9,710 $\times 10^{-12} \mathrm{~m}^{2} \mathrm{~s}^{-1}$ (Prasad y Prasad, 2013), de $10^{-9}$ a $10^{-10}$ en el secado de esta misma (Simha y Gugalia, 2013), en Amaranthus hybridus L. y Xanthosoma sagittifolium (L.) Schott, se reportaron $1,95 \times 10^{-9}$ y $2,09 \times 10^{-9} \mathrm{~m}^{2} \mathrm{~s}^{-1}$, respectivamente (Ako-

Cuadro 5.Variación de la difusividad efectiva del agua con el escaldado y la temperatura.

Table 5. Variation of the effective diffusivity of water with scalding and temperature.

\begin{tabular}{cccc}
\hline TEMP $\left({ }^{\circ} \mathbf{C}\right)$ & M0 & M1 & M2 \\
\hline 50 & $3,871^{-12} \mathrm{a}$ & $5,870^{-12} \mathrm{a}$ & $6,364^{-12} \mathrm{a}$ \\
60 & $7,340^{-12} \mathrm{~b}$ & $7,970^{-12} \mathrm{~b}$ & $7,087^{-12} \mathrm{~b}$ \\
70 & $9,763^{-12} \mathrm{c}$ & $1,175^{-11} \mathrm{c}$ & $1,097^{-11} \mathrm{c}$ \\
\hline
\end{tabular}

Los valores con letras diferentes indican que son significativamente diferentes $(\mathrm{P}<0,05)$ según el análisis de varianza y la comparación múltiple de Tukey. 
nor y Amankwah, 2012), y en tomillo de $3,69 \times 10^{-12}$ a $1,19 \times 10^{-10} \mathrm{~m}^{2} \mathrm{~s}^{-1}$ (Da Rocha et al., 2012). En cuanto a las variaciones presentadas entre las diferentes matrices alimentarias, Rao et al. (2005) indican que la presencia de grasas disminuye significativamente el proceso de difusividad del agua. Así, se deduce que el contenido de grasa en las hojas de quinua no varía con los pretratamientos, manteniendo valores de $D_{\text {eff }}$ bajos. Adicionalmente Ratti (2009) indicó que factores influyentes son la velocidad de circulación del aire de secado, los tipos de procedimientos, las condiciones experimentales para determinar los contenidos de agua, los métodos usados para el tratamiento de los datos obtenidos, y la heterogeneidad de las estructuras alimenticias.

La energía de activación fue calculada al graficar $\ln \left(D_{\text {eff }}\right)$ en función de la inversa de la temperatura (1/ $(\mathrm{T}+273,15))$ y se presentan en la Figura 7 y las ecuaciones (12) al (14) muestran el efecto de la temperatura sobre $D_{\text {eff }}$ en las muestras control y pre-tratadas como sigue:

M0 = Muestras control

$$
D_{\text {eff }}=3,38 * 10^{-5}\left(\frac{5147,59657}{T+273,15}\right)
$$

M1 = Muestras escaldado en agua

$$
D_{\text {eff }}=8,47 * 10^{-7}\left(\frac{3844,37028}{T+273,15}\right)
$$

M2 = Muestras escaldados en solución de $\mathrm{NaCl}$ al 3\%

$$
D_{\text {eff }}=6,48 * 10^{-8}\left(\frac{2999,98166}{T+273,15}\right)
$$

Los valores de $E_{a}$ son 56,$044 ; 41,855$ y $32,66 \mathrm{~kJ} \mathrm{~mol}^{-1}$ para M0, M1 y M2. Los valores de la energía de activación se encuentran dentro del rango general para los materiales alimenticios de 12,7-110,0 kJ mol-1 Doymaz (2014). Al respecto Darvishi et al. (2013) resaltan que los valores altos de $E_{a}$ indican una menor sensibilidad de la $D_{\text {eff }}$ a la temperatura. Los valores del presente estudio están por debajo de los valores reportados para tomillo 77,16 kJ mol${ }^{-1}$ (Da Rocha et al., 2012), hojas de menta $62,96 \mathrm{~kJ} \mathrm{~mol}^{-1}$ (Ratti, 2009), siendo más similares al valor reportado para espinaca $50,851 \mathrm{KJ} \mathrm{mol}^{-1}$ (Prasad y Prasad, 2013), y ligeramente superiores que para rodajas de tomate $22,28 \mathrm{KJ} \mathrm{mol}^{-1}$ (Abano et al., 2011), estos últimos indican que un proceso controlado por la difusión tiene una energía de activación menor $\sim 34 \mathrm{~kJ} \mathrm{~mol}^{-1}$, por lo tanto los valores estimados de $E_{a}$

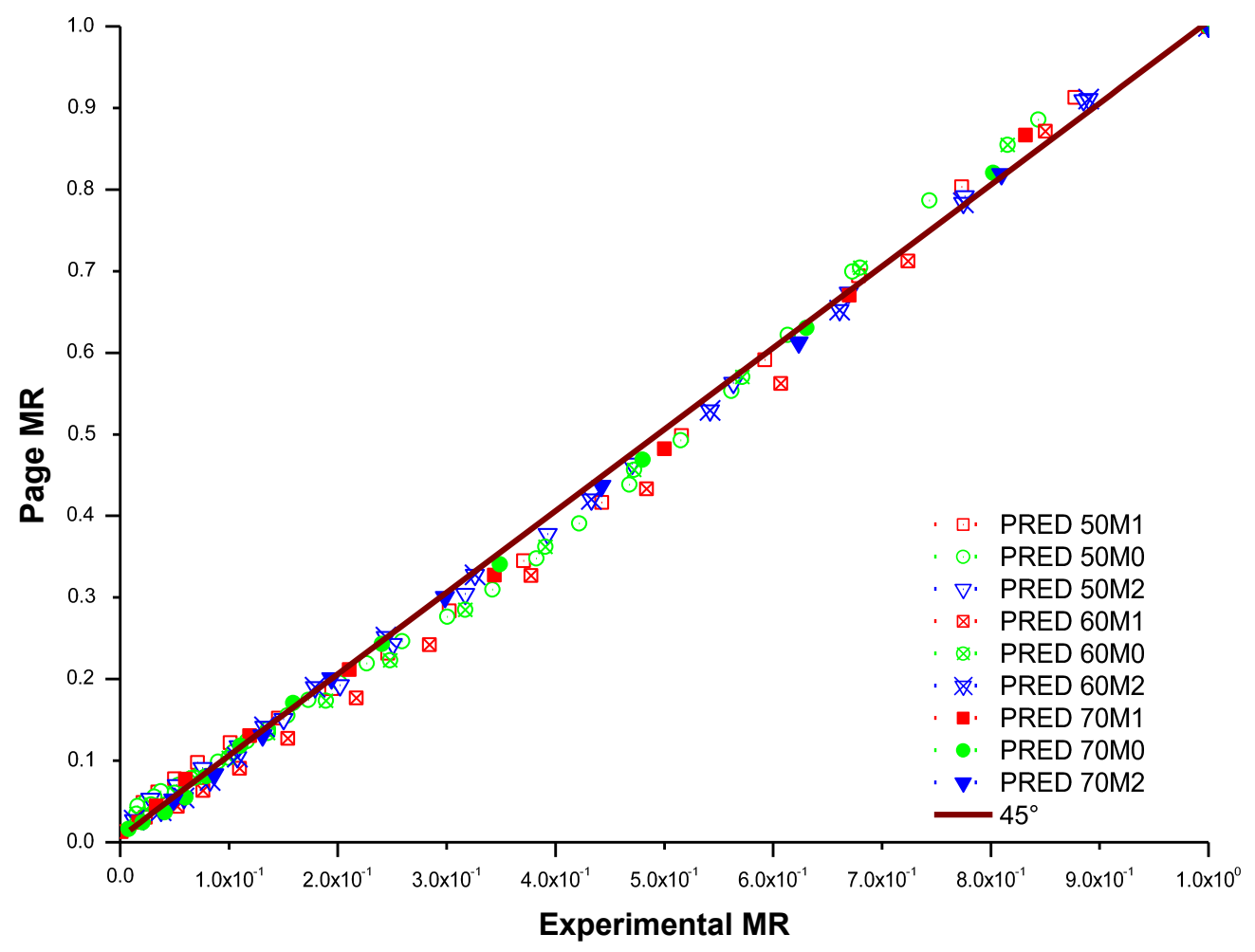

Figura 7. Relación entre la difusividad y el inverso de temperatura.

Figure 7. Relationship between the diffusivity and the inverse of temperature. 
para la presente investigación sugieren que la difusión del agua en las hojas de quinua es un mecanismo limitante para trabajar con M0. Sin embargo, en las muestras escaldadas con agua y $\mathrm{NaCl}$ a altas temperaturas no hay gran resistencia a la deshidratación por difusión por parte de las hojas de quinua particularmente en M2 que representa relativa baja demanda de energía.

El grado de rehidratación de los productos deshidratados es dependiente de los cambios estructurales y químicos que ocurren a nivel intrínseco (Akonor y Tortoe, 2014). La Figura 8 relaciona el coeficiente de difusión (eje Y) con los parámetros termodinámicos en base a la temperatura absoluta (eje X). La gráfica muestra una rápida absorción de agua al comienzo de la rehidratación como resultado de la absorción superficial y capilar que fue observada en los primeros 60 minutos en M1 y M2, pero en M0 la rehidratación es más lenta y prácticamente constante a partir de los 30 minutos en adelante.

En la Figura 9 se observa que la rehidratación de las muestras pre-tratadas M1 resultó con la mayor relación de rehidratación, seguida de las muestras M2, mientras que la muestra control resultó con valores más bajos. Esta observación, puede deberse al trastorno estructural de las células causado por el calor del agua de escaldado y el uso del $\mathrm{NaCl}$. Los resultados obtenidos muestran mejores niveles de rehidratación en comparación con muestras de Diospyros kaki L., y Agaricus bisporus (J.E. Lange), ambas secadas sin y con pre-tratamiento y rehidratadas por 240 minutos (Doymaz, 2012; Doymaz, 2014). Asimismo, mayores que para cubos de papa pre-tratados Severini et al. (2005).
Por otro lado, Akonor y Tortoe (2014) mencionan que para la reconstitución de productos deshidratados es deseable una alta estructura porosa de las matrices alimentarias.

\section{CONCLUSIONES}

Existe influencia de la temperatura del aire de secado y el pre-tratamiento aplicado sobre la reducción del tiempo de secado. El secado a velocidad decreciente fue registrado en la muestra control, y en las muestras escaldadas bajo las dos condiciones diferentes se observó velocidad constante. Al efectuar el ajuste de los datos experimentales con cinco modelos empíricos de la literatura y de acuerdo al análisis estadístico aplicado los modelos de Page y Midilli caracterizan el secado de hojas de quinua por presentar altos valores de $\mathrm{R}^{2}$ de 0,9995 - 0,9985 con valores de RMSE y $\chi^{2}$ bajos de $0,0053-0,016$ y $4,01 \times 10^{-5}-1,23 \times 10^{-4}$, respectivamente, en concordancia, estos resultados mostraron buena convergencia con los datos experimentales. La difusividad efectiva del agua varió de $3,871 \times 10^{-12}$ a $1,175 \times 10^{-11}$ $\mathrm{m}^{2} \mathrm{~s}^{-1}$ existiendo una clara influencia de la temperatura y los pre-tratamientos aplicados desde que la energía de activación varió de 32,66 a 56,044 kJ mol-1. Por otro lado, los contenidos de grasa, ceniza, fibra, proteína e hidratos de carbono son mejor retenidos cuando las hojas de quinua se escaldan en solución de $\mathrm{NaCl}$ al 3\% sin diferencias significativas $\mathrm{P}<0,05$ respecto al control. Mientras, las muestras escaldadas en agua presentaron los mejores índices de rehidratación $(4,75)$ y la muestra control los menores índices de rehidratación $(3,03)$.

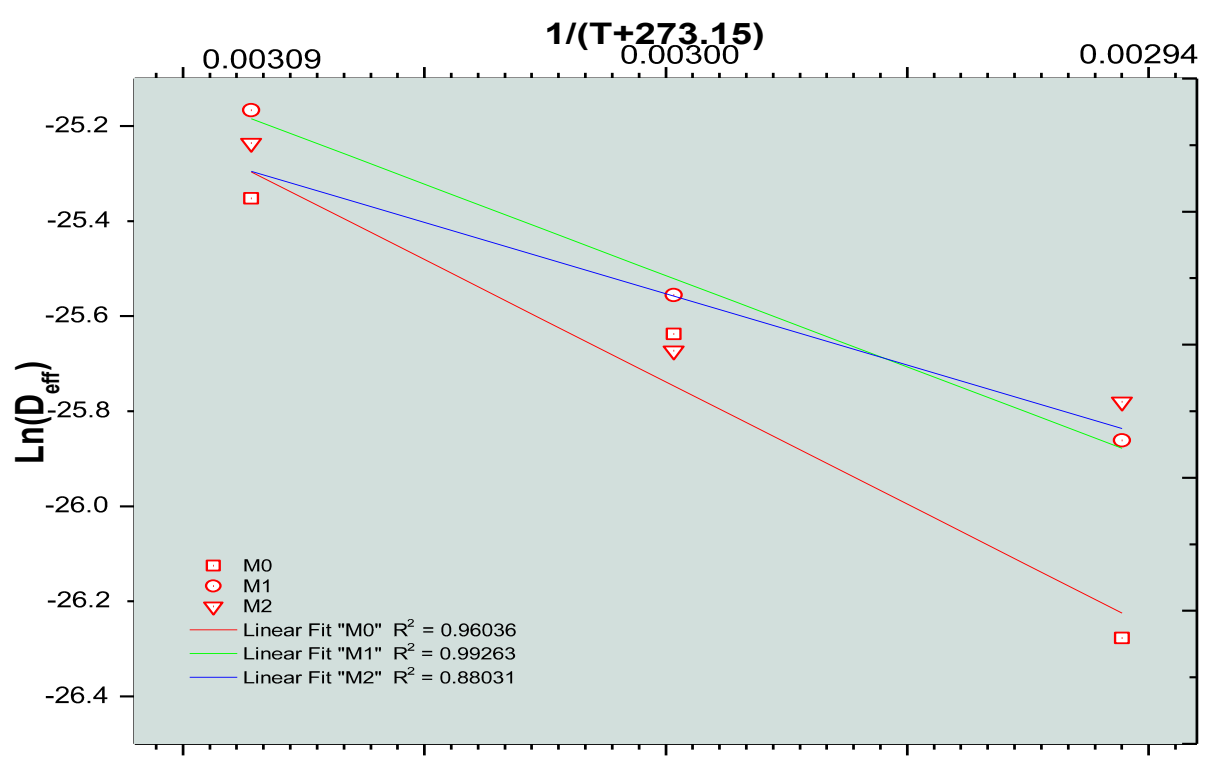

Figura 8. Relación de rehidratación en función del tiempo a $50^{\circ} \mathrm{C}$ de las muestras deshidratadas.

Figure 8. Rehydration ratio as a function of time at $50{ }^{\circ} \mathrm{C}$ of dehydrated samples. 


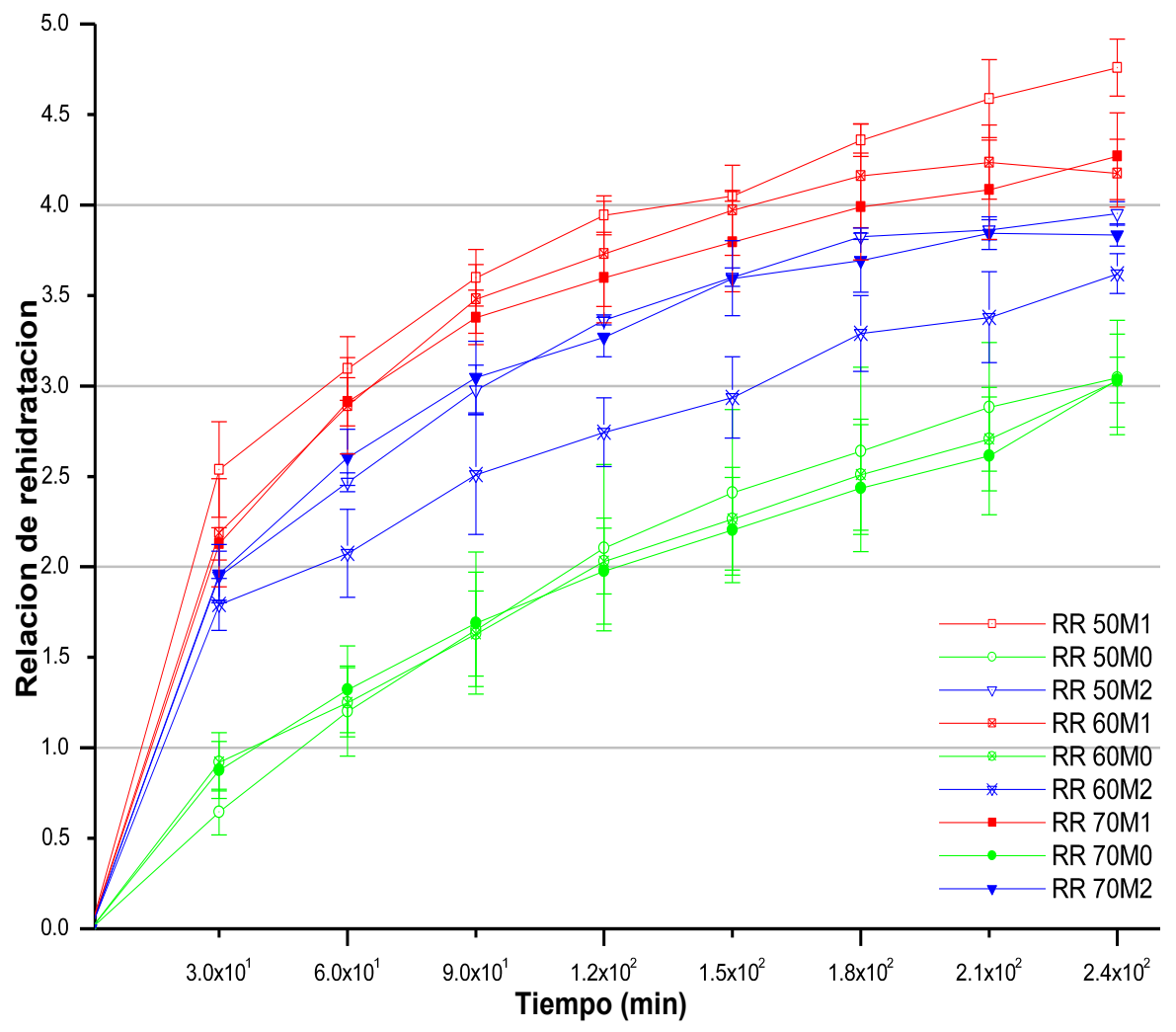

Figura 9. Relación de rehidratación de la muestras pre-tratadas en función del tiempo.

Figure 9. Rehydration ratio of pre-treated samples as a function of time.

\section{REFERENCIAS}

Abano, E.E., Ma, H., Qu, W., 2011. Influence of air temperature on the drying kinetics and quality of tomato slices. Journal of Food Processing \& Technology 2(5), 123. https:// doi.org/10.4172/2157-7110.1000123

Adolf, V., Jacobsen, S.-E., Shabala, S., 2013. Salt tolerance mechanisms in quinoa (Chenopodium quinoa Willd.). Environmental and Experimental Botany 92, 43-54.

Adnan Ramzani, P.M., Shan, L., Anjum, S., Din Khan, W., Ronggui, H., Iqbal, M., Abbas, Z., Kausar, S., 2017. Improved quinoa growth, physiological response, and seed nutritional quality in three soils having different stresses by the application of acidified biochar and compost. Plant Physiology and Biochemistry 116, 127-138. https://doi. org/10.1016/j.plaphy.2017.05.003

Agarry, S.E., Ajani, A.O., Aremu, M.O., 2013. Thin layer drying kinetics of pineapple: effect of blanching temperature time combination. Nigerian Journal of Basic and Applied Science 21(1), 1-10. http://dx.doi.org/10.4314/njbas. v21i1.1

Aghbashlo, M., Kianmehr, M.H., Khani, S., Ghasemi, M., 2009. Mathematical modelling of thin-layer drying of carrot. International Agrophysics 23(4), 313-317.

Akonor, P.T., Amankwah, E.A., 2012. Thin layer drying kinetics of solar-dried Amaranthus hybridus and Xanthosoma sagittifolium leaves. Journal of Food Processing \& Te- chnology 3(8), 174. http://dx.doi.org/10.4172/21577110.1000174

Akonor, P.T., Tortoe, C., 2014. Effect of blanching and osmotic pre-treatment on drying kinetics, shrinkage and rehydration of chayote (Sechium edule) during convective drying. British Journal of Applied Science \& Technology 4(8), 1215-1229. http://dx.doi.org/10.9734/ BJAST $/ 2014 / 4567$

Association of Official Analytical Chemists (AOAC), 1990. Official Methods of Analysis, 15th ed. AOAC, Inc., Arlington, Virginia, USA.

Ayorkor, O.N., 2009. Effects of lanching and dehydration methods on the quality of moringa leaf powder used as herbal green tea. Master of Science Thesis, Kwame Nkrumah University of Science and Technology, Ghana. $108 \mathrm{p}$.

Bayindirli, A., 2010. Enzymes in fruit and vegetable processing. Taylor \& Francis Group, CRC Press, USA.

Cardozo, A., Tapia, M., 1979. Valor Nutritivo, en: Tapia, M., Gandarillas, H., Alandia, S., Cardoso, A., Mujica, A. (Eds.), La quinua y la kañiwa: cultivos andinos. CIID, Oficina Regional para la América Latina, Bogotá, Colombia, pp. 149-182.

Crank, J., 1975. The Mathematics of Diffusion, 2nd ed. Oxford University Press, London, UK.

Da Rocha, R.P., Melo, E., Corbín, J.B., Berbert, P.A., Donzeles, S., Tabar, J.A., 2012. Cinética del secado de tomillo. Re- 
vista Brasileira de Engenharia Agrícola e Ambiental 16(6), 675-683. http://dx.doi.org/10.1590/S141543662012000600013

Darvishi, H., 2012. Mathematical modeling, moisture diffusion and energy consumption in thin layer drying of alfalfa. Middle-East Journal of Scientific Research 12(4), 511-516. https://doi.org/10.5829/idosi.mejsr.2012.12.4.1700

Darvishi, H., Asl, A., Asghari, A., Najafi, G., Gazori, H.A., 2013. Mathematical modeling, moisture diffusion, energy consumption and efficiency of thin layer drying of potato slices. Journal of Food Processing \& Technology 4(3), 215. http://dx.doi.org/10.4172/2157-7110.1000215

Doymaz, I., 2012. Evaluation of some thin-layer drying models of persimmon slices (Diospyros kaki L.). Energy Conversion and Management 56, 199-205. https://doi. org/10.1016/j.enconman.2011.11.027

Doymaz, I., 2014. Drying kinetics and rehydration characteristics of convective hot-air dried white button mushroom slices. Journal of Chemistry 2014, 1-8. http:// dx.doi.org/10.1155/2014/453175

Espinoza, J.L., 2011. Aplicacion de un proceso de secado asistido infrarrojo para la deshidratación del fruto de murtilla (Ugni molinae Turcz.). Tesis de grado Ingeniería de Alimentos, Universidad de Chile. 82 p.

Fellows, P.J., 2000. Food processing technology: principles and practices, second ed. Woodhead Publishing Ltd., CRC Press, England.

Fiallos-Jurado, J., Pollier, J., Moses, T., Arendt, P., Barriga-Medina, N., Morillo, E., Arahana, V., Torres, M., Goossens, A., Leon-Reyes, A., 2016. Saponin determination, expression analysis and functional characterization of saponin biosynthetic genes in Chenopodium quinoa leaves. Plant Science 250, 188-197. https://doi.org/10.1016/j. plantsci.2016.05.015

Galarza, R.S., 2010. Elaboración de una sopa instantánea a base de hoja quinua verde (Chennopudium quinoa Willd.) a dos temperaturas de secado, nueve formulaciones, utilizando dos tipos de empaques y cinco tiempos de almacenamiento. Tesis de grado Ingerieria Agroindustrial, Universidad Técnica de Cotopaxi, Latacunga, Ecuador. 150 p.

Gawlik-Dziki, U., 'Swieca, M., Sułkowski, M., Dziki, D., Baraniak, B., Czyz, J., 2013. Antioxidant and anticancer activities of Chenopodium quinoa leaves extracts - In vitro study. Food and Chemical Toxicology 57, 154-160. http:// dx.doi.org/10.1016/j.fct.2013.03.023

Geankoplis, C.J., 2006. Procesos de transporte y principios de procesos de separación, cuarta ed. Compañía Editorial Continental, México.

Jokić, S., Velić, D., Bilić, M., Lukinac, J., Planinić, M., Bucić-Kojić, A., 2009. Influence of process parameters and pretreatments on quality and drying kinetics of apple samples. Czech Journal of Food Science 27(2), 88-94.

Jongen, W., 2002. Fruit and vegetable processing: improving quality. Woodhead Publishing Limited, Cambridge, England.

Karam, M.C., Petit, J., Zimmer, D., Djantou, E.B., E., Scher, J., 2016. Effects of drying and grinding in production of fruit and vegetable powders: A review. Journal of Food Engineering 188, 32-49. https://doi.org/10.1016/j. jfoodeng.2016.05.001

Kozioł, M.J., 1992. Chemical composition and nutritional evaluation of quinoa (Chenopodium quinoa Willd.). Journal of Food Composition and Analysis 5(1), 35-68. https:// doi.org/10.1016/0889-1575(92)90006-6

López, L.M., Capparelli, A., Nielsen, A.E., 2011. Traditional post-harvest processing to make quinoa grains (Chenopodium quinoa var. quinoa) apt for consumption in Northern Lipez (Potosí, Bolivia): ethnoarchaeological and archaeobotanical analyses. Archaeological and Anthropological Sciences 3(1), 49-70. https://doi. org/10.1007/s12520-011-0060-5

Lorusso, A., Verni, M., Montemurro, M., Coda, R., Gobbetti, M., Rizzello, C.G., 2017. Use of fermented quinoa flour for pasta making and evaluation of the technological and nutritional features. LWT - Food Science and Technology 78, 215-221. https://doi.org/10.1016/j. lwt.2016.12.046

Masterbroek, H.D., Limburg, H., Gilles, T., Marvin, H.J.P., 2000. Occurrence of sapogenins in leaves and seeds of quinoa (Chenopodium quinoa Willd.). Journal of the Science of Food and Agriculture 80(1), 152-156. https://doi.org/10.1002/(SICI)10970010(20000101)80:1<152::AID-JSFA503>3.0.CO;2-P

Meisami-asl, E., Rafiee, S., 2009. Mathematical modeling of kinetics of thin-layer drying of apple (var. Golab). Agricultural Engineering International XI, 1185.

Mepba, H.D., Eboh, L., Banigo, D.E.B., 2007. Effects of processing treatments on the nutritive composition and consumer acceptance of some Nigerian edible leafy vegetables. African Journal of Food, Agriculture, Nutrition and Development 7(1), 1-18.

Midilli, A., Kucuk, H., Vapar, Z., 2002. A new model for singlelayer drying. Drying Technology 20(7), 1503-1513. https://doi.org/10.1081/DRT-120005864

Motevali, A., Younji, S., Chayjan, R.A., Aghilinategh, N., Banakar, A., 2013. Drying kinetics of dill leaves in a convective dryer. International Agrophysics 27(1), 39-47. https://doi.org/10.2478/v10247-012-0066-y

Mujica, A., Jacobsen, S., 2006. La quinua (Chenopodium quinoa Willd.) y sus parientes silvestres, en: Moraes, M., Ollgaard, B., Kvist, L.P., Borchsenius, F., Balslev, H. (Eds.), Botánica Económica de los Andes Centrales. Universidad Mayor de San Andrés, La Paz, pp. 449-457.

Navruz-Varli, S., Sanlier, N., 2016. Nutritional and health benefits of quinoa (Chenopodium quinoa Willd.). Journal of Cereal Science 69, 371-376. https://doi.org/10.1016/j. jcs.2016.05.004

Nowak, V., Du, J., Charrondiere, R., 2016. Assessment of the nutritional composition of quinoa (Chenopodium quinoa Willd.). Food Chemistry 193, 47-54.

Olurin, T.O., Adelekan, A.O., Olosunde, W.A., 2012. Mathematical modelling of drying characteristics of blanched field pumpkin (Cucurbita pepo L.) slices. Agricultural Engineering International: CIGR Journal 14(4), 246-254.

Organización de las Naciones Unidades para la Alimentación y la Agricultura (FAO), 2013. Lanzamiento del año internacional de la Quinua. http://www.fao.org/americas/ noticias/ver/es/c/229953/ (acceso, 19.02.2013).

Prasad, A., Prasad, K., 2013. Studies on kinetics of moisture removal from spinach leaves. International Journal of 
Agriculture and Food Science Technology 4(4), 303-308.

Rao, M.A., Rizvi, S.S.H., Datta., A.K., 2005. Engineering properties of foods, third ed. Taylor \& Francis Group, Boca Raton.

Ratti, C., 2009. Advances in food dehydration (Vol. 5). CRC Press, New York.

Regional Office for Latin America and the Caribbean (PROINPA), 2011. Quinoa: an ancient crop to contribute to world food security. $37^{\text {th }}$ FAO Conference, Bolivia. http://www.fao.org/docrep/017/aq287e/aq287e.pdf

Repo-Carrasco, R., Hellström, J.K., Pihlava, J., Mattila, P.H., 2010. Flavonoids and other phenolic compounds in Andean indigenous grains: quinoa (Chenopodium quinoa), kañiwa (Chenopodium pallidicaule) and kiwicha (Amaranthus caudatus). Food Chemistry 120(1), 128-133. https://doi.org/10.1016/j.foodchem.2009.09.087

Reyes, J., Cavalieri, R., Powers, J., 2004. Blanching of Foods. In: Dekker, M. (Ed.), Encyclopedia of Agricultural, Food, and Biological Engineering. Washington State University, Pullman, Washington, USA, pp. 1-5.

Saeed, I.E., Sopian, K., Zainol Abidin, Z., 2008. Thin-layer drying of roselle (I): Mathematical modeling and drying experiments. Agricultural Engineering International: the CIGR Ejournal X, 1-25.

Schlick, G., 2000. Nutritional characteristics and biomass production of Chenopodium quinoa grown in controlled environments. Master of Science Thesis, San Jose State University, USA. 35 p.

Severini, C., Baiano, A., De Pilli, T., Carbone, B.F., Derossi, A., 2005. Combined treatments of blanching and dehydration: study on potato cubes. Journal of Food Engineering 68(3), 289-296. https://doi.org/10.1016/j.jfoodeng.2004.05.045

Silva, A.S., Almeida, F., Lima, E.E., Silva, F., Gomes, J.P., 2008. Drying kinetics of coriander (Coriandrum sativum) leaf and stem. Ciencia y Tecnología Alimentaria 6(1), 13-19. https://doi.org/10.1080/11358120809487622

Simha, P., Gugalia, A., 2013. Thin layer drying kinetics and modelling of Spinacia oleracea leaves. International Journal of Applied Engineering Research 8(9), 1053-1066.

Sonmete, M.H., Menges, H.O., Ertekin, C., Özcan, M.M., 2017. Mathematical modeling of thin layer drying of carrot slices by forced convection. Journal of Food Measurement and Characterization 11(2), 629-638. https://doi. org/10.1007/s11694-016-9432-y

Sutar, N., Sutar, P.P., 2013. Developments in osmotic dehydration of fruits and vegetable: a review. Trends in Post Harvest Technology 1(1), 20-36.

Świeca, M., Sęczyk, L., Gawlik-Dziki, U., Dziki, D., 2014. Bread enriched with quinoa leaves - The influence of proteinphenolics interactions on the nutritional and antioxidant quality. Food Chemistry 162, 54-62. https://doi. org/10.1016/j.foodchem.2014.04.044

Taheri-Garavand, A., Rafiee, S., Keyhani, A., 2011. Mathematical modeling of thin layer drying kinetics of tomato influence of air dryer conditions. International Transaction Journal of Engineering, Management, \& Applied Sciences \& Technologies 2, 147-160. http://TuEngr. com/V02/147-160.pdf

Torregroza-Espinosa, A.M., Montes-Montes, E.J., Ávila-Gómez, A.E., Remolina-López, J.F., 2014. Modelado de las cinéticas de secado de tres variedades de yuca industrial. DYNA 81, 184-192.

Upadhyaya, A.K., Gupta, B., Garg, S., Singh, M., Pandey, M., 2012. Mathematical modeling of hot air drying of spinach leaves in universal hot air oven. International Journal of Advanced Research in Computer Engineering \& Technology 1(4), 153-157.

Veda, S., 2009. Bioavailability of $\beta$-carotene as influenced by food processing and presence of factors such as spices. Thesis in Doctor of Philisophy Thesis, Central Food Technological Research Institute, Mysore, India. 234 p.

Vilcacundo, R., Hernández-Ledesma, B., 2017. Nutritional and biological value of quinoa (Chenopodium quinoa Willd.). Current Opinion in Food Science 14, 1-6.

Villacrés, E., Peralta, E., Egas, L., Mazón, N., 2011. Potencial agroindustrial de la quinua. Boletín Divulgativo $\mathrm{N}^{\circ} 146$, Instituto Nacional Autónomo de Investigaciones Agropecuarias (INIAP), Quito, Ecuador, pp. 1-32. http://quinua.pe/potencial-agroindustrial-de-la-quinua/

Zhang, R., Long, J., 2017. Study on drying uniformity of static small-sized drying box for fruits and vegetables. Procedia Engineering 205, 2615-2622. https://doi. org/10.1016/j.proeng.2017.10.201 
